\title{
Electrons at the Surface of Quantum Systems
}

\author{
P. Leiderer
}

Fakultät für Physik, Universität Konstanz, Konstanz, Germany

Electrons can be trapped at the surfaces and interfaces of the condensed phases of quantum matter (in particular hydrogen and helium), where they form classical two-dimensional Coulomb systems. Apart from studying the intrinsic properties of these nearly ideal systems, like the transition from an electron gas to a Wigner solid, one can use the electrons also as a sensitive probe to investigate the surface of quantum liquids and solids. The examples presented here include the surface of solid $\mathrm{H}_{2}$, He films, and the interface between liquid and solid He, where phenomena such as annealing, layering, and crystal growth are studied. In addition, the interaction between the electrons and longwavelength interfacial modes leads to an electrohydrodynamic instability, which bears interesting similarities with other critical phenomena.

\section{INTRODUCTION}

The two lightest elements, hydrogen and helium, provide unique systems to study surfaces and interfaces of condensed matter in the presence of large quantum fluctuations. Surface phenomena to be mentioned here are, e.g., surface melting, roughening and layering. ${ }^{1}$ Since in the quantum systems the gas density above the surface is relatively high in the regions of interest, investigations using conventional surface techniques like LEED and EELS are of limited applicability, because they usually require high vacuum conditions. A technique which has been applied successfully is neutron scattering, which-although not being particularly sensitive to surfaces-can be used if the quantum systems are adsorbed on substrates with a large intrinsic surface area, like exfoliated graphite. ${ }^{2}$ Information about quantum surfaces has been obtained also from measurements of specific heat, adsorption isotherms, third-sound propagation ${ }^{19-22}$ and, as a somewhat peculiar method, from the investigation of surface state electrons (SSE). It is the latter which is the topic of this paper. 
Electrons near the surface of a dielectric are attracted due to the image potential

$$
V(z)=\left(\frac{1}{z}\right) e^{2} \frac{(\varepsilon-1)}{4(\varepsilon+1)} .
$$

Here $e$ is the elementary charge, $\varepsilon$ is the dielectric constant of the material, and $z$ is the distance of the electron from the surface. For the condensed phases of hydrogen and helium this attraction is rather weak because of their low polarizability. In addition, at atomic distances the negative electron affinity of $\mathrm{H}_{2}$ and $\mathrm{He}$ leads to a potential barrier, which is $\sim 10^{4} \mathrm{~K}$ for $\mathrm{He}^{3}$ and $\sim 3 \times 10^{4} \mathrm{~K}$ for $\mathrm{H}_{2}{ }^{4}$ As a result the electrons are trapped in a potential well perpendicular to the surface, forming states similar to the electronic radial distribution in a hydrogen atom, however with a much smaller binding energy in the ground state $\left(8 \mathrm{~K}\right.$ for $\mathrm{He}, 160 \mathrm{~K}$ for $\mathrm{H}_{2}$ ) and a much larger effective Bohr radius $\left(a_{B}=76 \AA\right.$ for $\mathrm{He}, 17 \AA$ for $\left.\mathrm{H}_{2}\right)$.

In contrast to the quantization perpendicular to the surface, the electrons can move parallel to the surface as nearly free particles, provided the substrate is smooth enough. An example where this condition is fulfilled in an almost ideal way is the surface of liquid helium (see Fig. 1), where at low temperatures the motion of individual electrons is only limited by scattering from quantized surface waves ("ripplons"). The two-dimensional (2-D) electron systems which can thus be generated on liquid helium are a classical counterpart to the degenerate 2-D electron system on MOSFET and related semiconductor structures.

The reason for classical statistics to apply for the surface state electrons on liquid helium is that the electron density obtainable is limited to $n<n_{\text {crit }}=$ $2.4 \times 10^{9} \mathrm{~cm}^{-2}$ (see below). This corresponds to a Fermi temperature of only $50 \mathrm{mK}$, much lower than the actual temperature in most of the experiments up to now.

A number of review articles has been devoted to the system of electrons on liquid helium, ${ }^{3}$ and some of the most important results are: (i) the excited electronic states are hydrogen-like, and spectroscopic measurements in the range of a hundred $\mathrm{GHz}$ agree well with calculations; (ii) the mass of the SSE is close to the free electron mass, as measured, for example, by cyclotron resonance; (iii) Coulomb interaction in the 2-D layer of electrons leads to collective excitations, i.e. 2-D longitudinal plasmons; (iv) one of the most intriguing effects in this system is the formation of an electron solid, the so-called Wigner crystal, which appears when the plasma parameter (the ratio of Coulomb and thermal energy) rises above about $130 .^{5}$

It would be extremely interesting to investigate the Wigner crystal also at densities higher than the above-mentioned limit $n_{\text {crit }}$ and trace out the complete phase diagram, in particular the melting of the crystal towards 


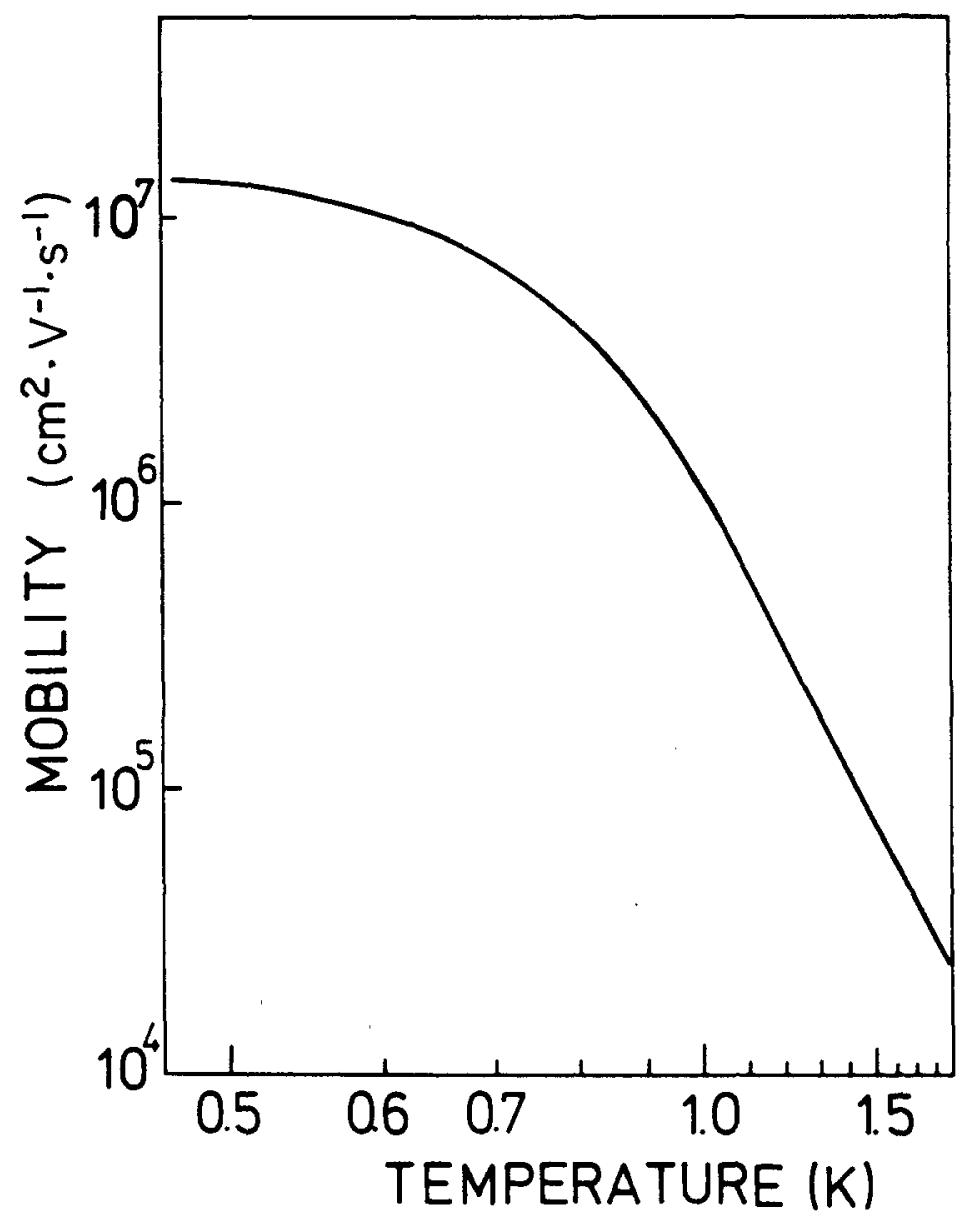

Fig. 1. Electron mobility on bulk ${ }^{4} \mathrm{He}$. The dominant electron scattering mechanisms are scattering from ripplons and gas atoms at low and high temperature, respectively (after ref. 3 ).

the degenerate Fermi liquid at low $T$ and the vicinity of the critical point which is expected above $10 \mathrm{~K}$. Unfortunately, these regions are not accessible with liquid helium as a substrate, because there an electrohydrodynamic (EHD) instability develops at $n_{\text {crit }}$ (see Sec. 3). ${ }^{6,7}$

Two routes have been pursued to circumvent the EHD instability. If the electrons are localized on a thin helium film rather than on bulk liquid, a density of up to $10^{11}$ electrons $/ \mathrm{cm}^{2}$ could be obtained due to stabilization of the film by van der Waals forces. A second possibility is to use a solid 
substrate like $\mathrm{H}_{2}$, and also in this case the experimental maximum electron density could be increased by more than an order of magnitude. As a disadvantage of these substrates, the mobility of the SSE was reduced considerably compared with the high value on bulk helium due to interaction with surface imperfections. In the course of the experiments it turned out, however, that this originally undesirable effect can be used as a very sensitive method to study surfaces by means of SSE.

Electrons can also be applied in another form for investigating quantum surfaces and interfaces: If they have high enough energy, they can overcome the potential barrier at the surface and penetrate into the condensed phase, where they rapidly relax into electron bubbles with a diameter of some $20 \AA$ (depending on pressure) and consequently an effective mass six orders of magnitude above the mass of free electrons. At low enough temperatures these "negative ions" can be trapped at the liquid helium surface from underneath, again forming a classical 2-D Coulomb system. In addition, the electron bubbles can be trapped at the interface between liquid and solid helium, where they can be used to study melting and crystallization under very clean conditions. ${ }^{8}$

In the following, I shall first discuss in Sec. 2 SSE as a probe for investigating surface phenomena like layering, annealing, and the relaxation of crystal surfaces. In these examples the surface charges are applied at such low densities that they do not appreciably influence the surface under study. As a second topic, Sec. 3 will treat the effects of high charge densities on the behavior of the surface, such as ripplon softening and various instability phenomena.

\section{SURFACE STATE ELECTRONS AS A SURFACE PROBE}

\subsection{Electrons on Solid $\mathrm{H}_{2}$}

Experiments with electrons on bulk solid $\mathrm{H}_{2}$ were first performed by Troyanovskii et al., ${ }^{10,11}$ who measured the ac conductivity of the SSE at frequencies in the $\mathrm{MHz}$ range. The crystal was grown in this case from the liquid phase at the triple point. A similar preparation procedure was used by Edel'man and Faley ${ }^{12}$ for cyclotron resonance studies of the SSE at $20 \mathrm{GHz}$, and by Cieslikowski et al. ${ }^{13.14}$ for mobility measurements in the $\mathrm{kHz}$ range. In all these experiments a characteristic temperature dependence was obtained for the electron mobility, as shown in Fig. 2.

At temperatures above about $10 \mathrm{~K}$ one important mechanism for energy loss of the electrons is gas atom scattering, which in dense gas (i.e. at the highest temperatures) is further increased by the tendency for the formation of electron bubbles. ${ }^{15}$ A second contribution arises from scattering by surface 


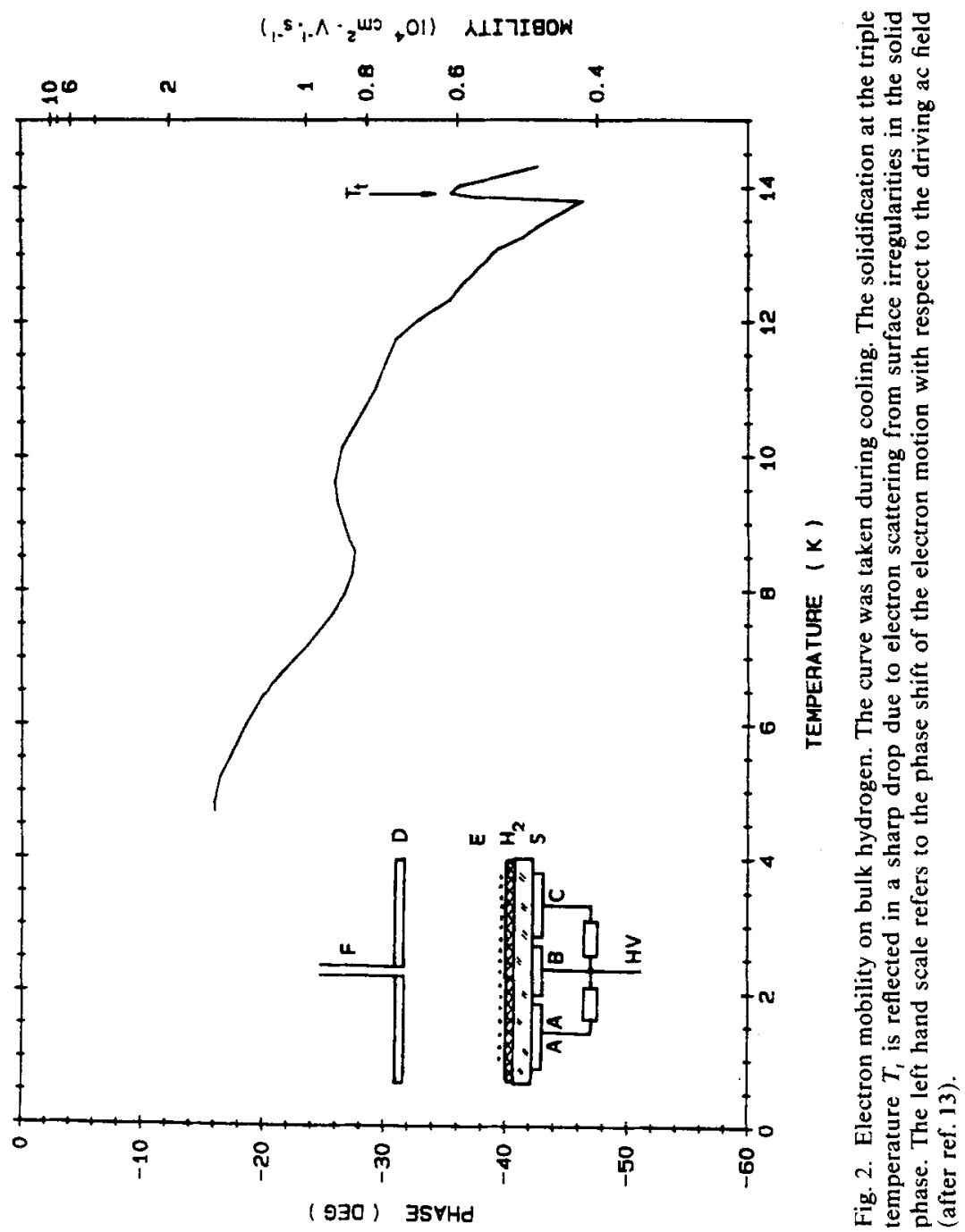


roughness, which appears as soon as the solid is formed, showing up in Fig. 2 as a sharp step at the triple temperature $T_{t}=13.9 \mathrm{~K}$. The height of this step varies with the parameters of the growth process and apparently depends on the surface quality of the crystal.

At $T \leq \mathrm{K}$ gas atom scattering becomes extremely small, and hence the principal scattering mechanism is due to surface defects. (The contribution of scattering from surface excitations on the solid (Rayleigh waves), as calculated by Troyanovskii et al., ${ }^{11}$ is more than two orders of magnitude below the experimental data and hence is negligible here. Likewise, the system of electrons is not noticeably influenced by the ortho-para conversion of hydrogen.) From an analysis of their mobility data between 4 and $10 \mathrm{~K}$ Troyanovskii $e t$ al. conclude that the main surface imperfections responsible for the scattering are defects with a dimension on the order of the lattice constant and a density of $2 \times 10^{12} \mathrm{~cm}^{-2}$. Another model for the surface defects was considered by Edel'man and Faley, ${ }^{12}$ who supposed that their solid hydrogen surfaces had a terrace structure with flat sections about $10^{-5} \mathrm{~cm}$ in size and steps much larger than an atomic distance. In confirmation of the estimate of rather high steps results for the same $\mathrm{H}_{2}$ surfaces covered with a superfluid ${ }^{4} \mathrm{He}$ film of $200 \AA$ thickness were presented. It was argued that if the scattering centres had atomic distances such a film should yield a substantial increase of the electron mobility up to the value known from bulk helium. Since this was not observed, it was inferred that the surface imperfections had at least a height comparable with the film thickness.

As reported by Edel'man and Faley and also by Cieslikowski et al., ${ }^{13}$ irregular variations of the electron mobility were observed quite frequently for temperatures around $8 \mathrm{~K}$. A slight indication of these effects is also visible in Fig. 2. It was suggested by Edel'man that this might be a signature of a roughening transition, ${ }^{12}$ although bulk effects like cracks resulting from differences in the thermal expansion of $\mathrm{H}_{2}$ crystal and sample cell also appear possible.

Whereas all the $\mathrm{H}_{2}$ crystal surfaces discussed so far had been grown from the liquid phase (with a crystal thickness $d \geq 1 \mathrm{~mm}$ ), more recently also thin $\mathrm{H}_{2}$ films (thickness $\sim 1 \mu \mathrm{m}$ ), quench-condensed at $T=1.5 \mathrm{~K} \ll T_{t}$, were investigated with SSE by Kono et al. ${ }^{16}$ Such films might be an interesting substrate for 2-D electron systems, since they allow to reach high electron densities already at low holding voltages.

In the experiments of Kono et al. it was observed that SSE on the freshly prepared films displayed an extremely low conductivity $\sigma$, apparently due to a very rough surface. However, $\sigma$ could be increased by orders of magnitude by thermal annealing of the films. As an example, Fig. 3 shows data for a $\mathrm{H}_{2}$ film treated in subsequent annealing steps. It was found that 


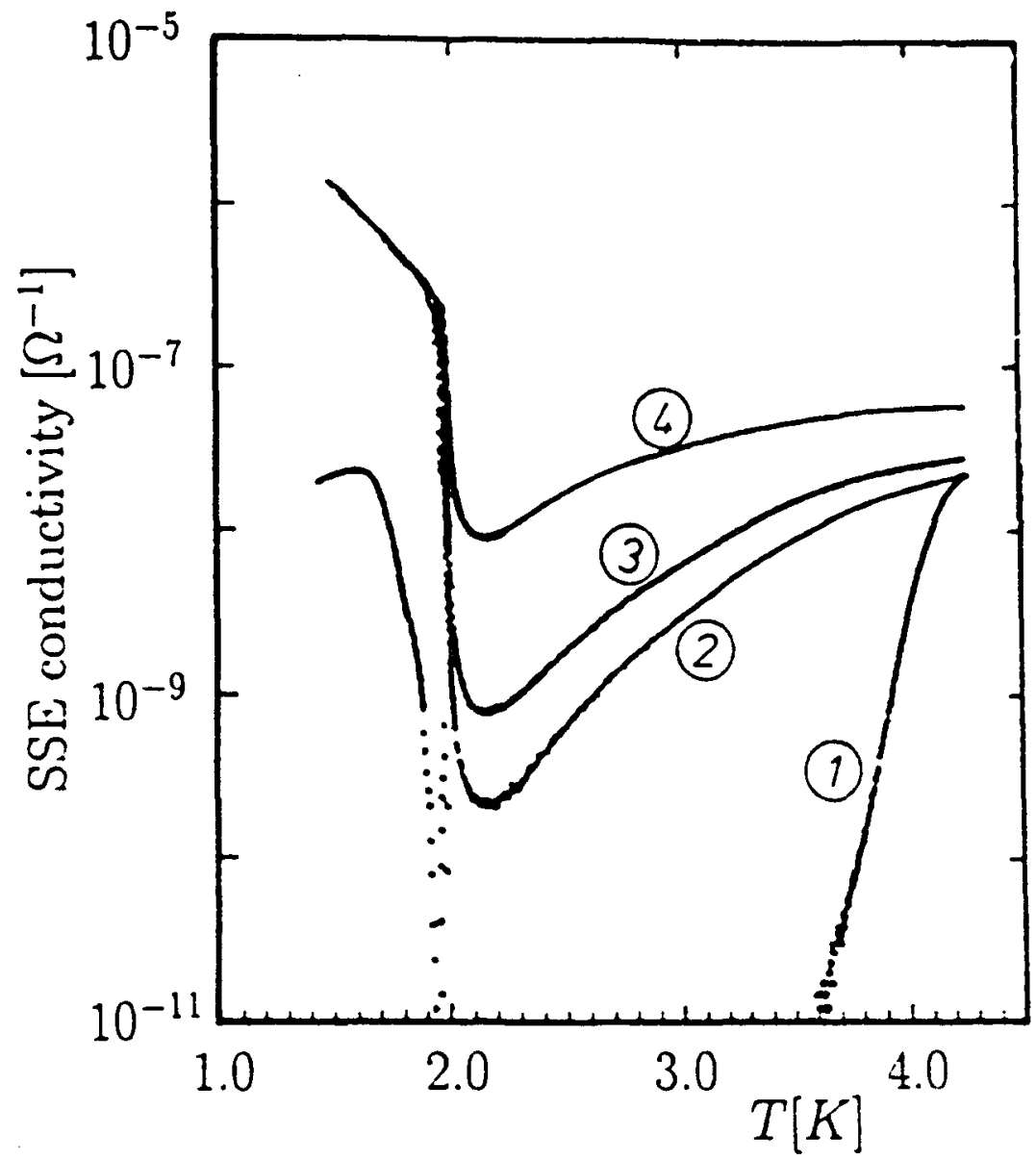

Fig. 3. Electron conductivity on a quench-condensed hydrogen film for various annealing steps: 1 -as prepared at $1.5 \mathrm{~K} ; 2$-briefly annealed at $4.2 \mathrm{~K} ; 3$-annealed for $12 \mathrm{~h}$ at $4.2 \mathrm{~K} ; 4$-briefly annealed at $8 \mathrm{~K}$. In addition to the hydrogen a small amount of helium was admitted to the cell, which formed a saturated film of about $300 \AA$ thickness below $T \approx 1.9 \mathrm{~K}$, giving rise to a steep increase in the conductivity. The electron density was $4 \times 10^{8} \mathrm{~cm}^{-2}$ in this case (ref. 16).

$\sigma$ could be described approximately by an Arrhenius law* $\sigma=$ $\sigma_{0} \exp (-E / k T)$, with an activation energy $E$ which decreased from 23 to $10 \mathrm{~K}$ in the course of the annealing procedure. The temperature dependence of $\sigma$ was interpreted as thermal activation of electrons trapped by surface

*For the data in Fig. 3 this applies only to temperatures $T \geq 2 \mathrm{~K}$, because in this experiment at smaller $T$ the $\mathrm{H}_{2}$ surface was covered with a helium film. 
irregularities; the decreasing activation energy then is an indication that upon annealing the traps become shallower and that therefore the degree of roughness of the surface decreases. Although a quantitative analysis is still lacking, the measurement of $\sigma$ appears as a promising tool to study surface conditions. It should be added that the films were completely smooth as judged by optical inspection, i.e. roughness on the scale of the wavelength of light could not be detected. Additional information was obtained from measurements with the $\mathrm{H}_{2}$ surface being covered with an additional film of superfluid ${ }^{4} \mathrm{He}$, similar to the experiments of Edel'man and Faley on bulk $\mathrm{H}_{2}{ }^{12}$ In contrast to these earlier results, however, the $\mathrm{He}$ film was found to have a drastic influence on the conductivity (see Fig. $3, T \leq 1.9 \mathrm{~K}$ ). In fact, for saturated $\mathrm{He}$ films the value for $\sigma$ coincided with the conductivity on bulk He (except for the as-quenched films, where it was lower by about a factor of 10), indicating that the amplitude of the surface roughness of the $\mathrm{H}_{2}$ films was distinctly smaller than the He film thickness of $300 \AA$.

\subsection{Electrons on Helium Films}

Electrons on saturated helium films with a typical thickness of 200 $400 \AA$ have already been mentioned briefly in the previous section. Now I discuss this system in more detail, first very thin He films in the monolayer range, then the transition region with a thickness of some ten monolayers and finally the saturated films of about a hundred monolayers. The isotope investigated was nearly exclusively ${ }^{4} \mathrm{He}$. The substrate for the helium was in general again solid $\mathrm{H}_{2}$, except for the films with several hundred $\AA$ thickness, where also results for more conventional dielectrics like glass or polymers exist. Neon substrates, which are in many respects similar to hydrogen, have been reviewed by Kajita. ${ }^{17}$

\section{Thin He Films}

One motivation for coating a hydrogen surface with helium was the idea that a He film on the order of a monolayer might heal the defects on the hydrogen. SSE on such films were investigated by Paalanen and Iye ${ }^{18}$ from 1.25 to $4.2 \mathrm{~K}$, and down to lower temperatures by Cieslikowski $e t$ al. ${ }^{13,14}$ These measurements showed that a $\mathrm{He}$ film did not improve the electron mobility $\mu$, as one might have expected on the basis of the above argument, but that on the contrary $\mu$ was reduced in the presence of helium. Furthermore, as the He film thickness was increased, the mobility did not drop monotonously but showed a pronounced oscillatory behavior. Mobility maxima occurred whenever the coverage reached an integral number of monolayers, whereas minima were observed at approximately half-filled topmost layers. This is illustrated in Fig. 4, taken from an experiment where 


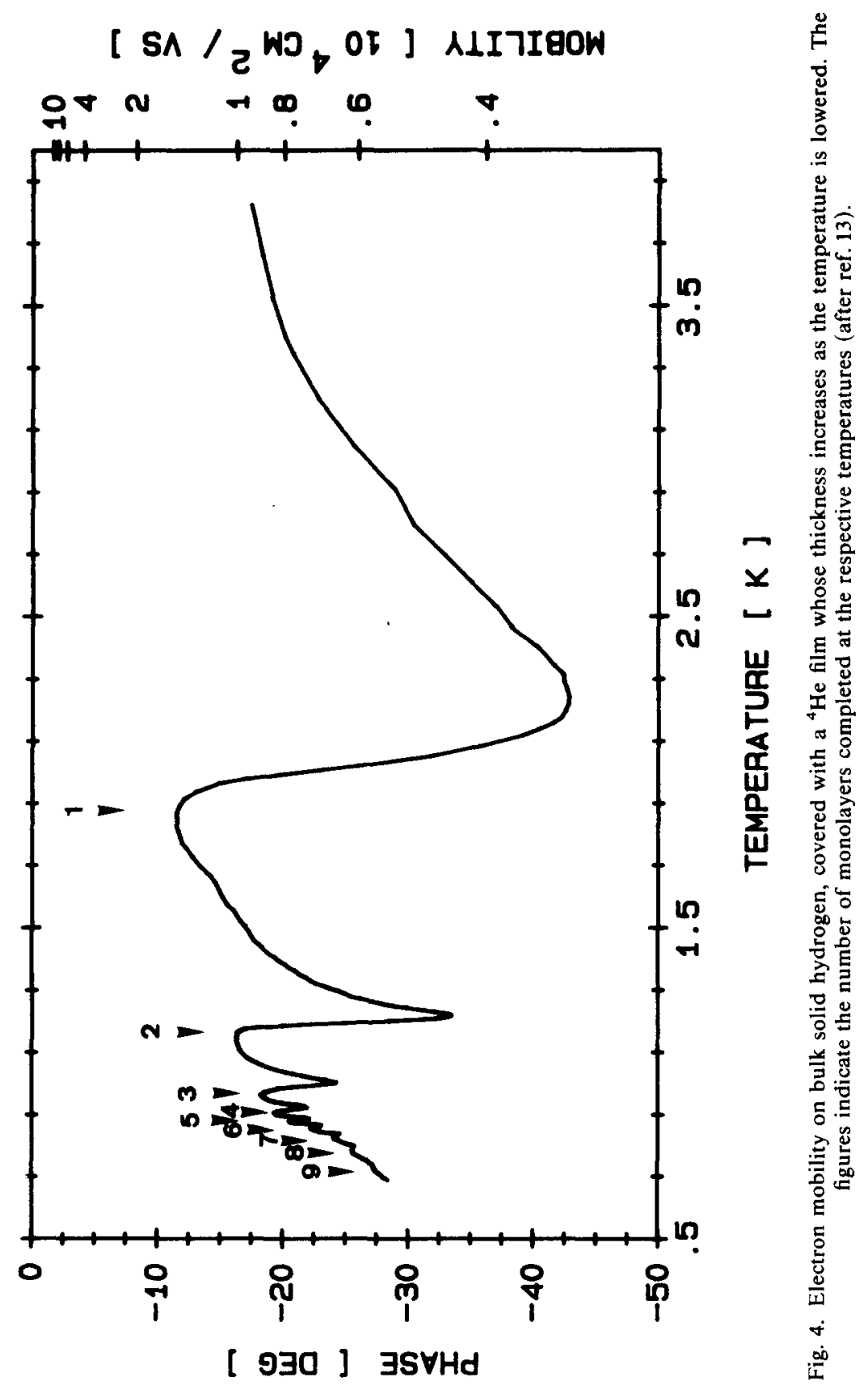


the film thickness was varied by changing the temperature at a fixed total amount of helium (gas phase + adsorbed film) in the cell.

For coverages in the submonolayer regime the increase in electron scattering was interpreted by Paalanen and Iye as being due to density fluctuations in the 2-D system of adsorbed helium atoms, which are proportional to the compressibility of this system. For a densely packed, complete monolayer of $\mathrm{He}$ on $\mathrm{H}_{2}$ the compressibility becomes small and hence the mobility reached nearly the value on the bare $\mathrm{H}_{2}$ substrate. For thicker films, qualitatively the same picture should apply for the topmost layer, as long as well-defined layers exist. The mobility signal therefore provides a measure of the extent of layering of the helium film. From the fact that nine mobility maxima could be resolved in the experiment by Cieslikowski et al. at temperatures below $0.8 \mathrm{~K}$ one can conclude that the layering effects in liquid helium* near a solid wall extent to at least nine interatomic distances. At higher $T$ they become more and more washed out.

Pronounced layering of $\mathrm{He}$ films, although not over quite as large a distance, has been observed also in several other experiments using specific heat, third sound, and adsorption isotherms. ${ }^{19-22}$ An unexpected result was reported recently by Shirron et l. $^{21}$ who observed modulations in the third-sound velocity with a periodicity distinctly smaller than a monolayer. However, in another third-sound experiment Mistura et al. ${ }^{22}$ found good agreement between the location of maxima in the third-sound velocity and the completion of monolayers as determined from adsorption isotherms. A formation of layers was also obtained in numerical simulations by Krotscheck. $^{23}$

\section{He Films at Medium Thickness}

In the intermediate region between monolayers and thick saturated films the mobility eventually starts to rise, because the image potential gradually decreases and approaches the value of bulk helium. Consequently also the interaction of the electrons with defects and surface excitations is reduced. This behavior, already shown in Fig. 3, is in some more detail illustrated in Fig. 5, where the transition region is plotted on an expanded scale. Strikingly the transition is not smooth, but a sharp dip is observed around a film thickness of 10 monolayers with a half width of only 1-2 monolayers. $\uparrow$ This dip turns out to be a rather general phenomenon, because not only has it been observed for all the quench-condensed $\mathrm{H}_{2}$ substrates,

\footnotetext{
*It is known from other substrates that helium usually forms one or two solid layers next to a wall, caused by the van der Waals pressure. For $\mathrm{H}_{2}$, due to its small interaction with $\mathrm{He}$, it is not clear yet whether the first layer of He solidifies.

tThe structure due to layering as discussed in the previous section does not appear in this figure because of the relatively high temperature and possibly also because of the roughness of the substrate.
} 


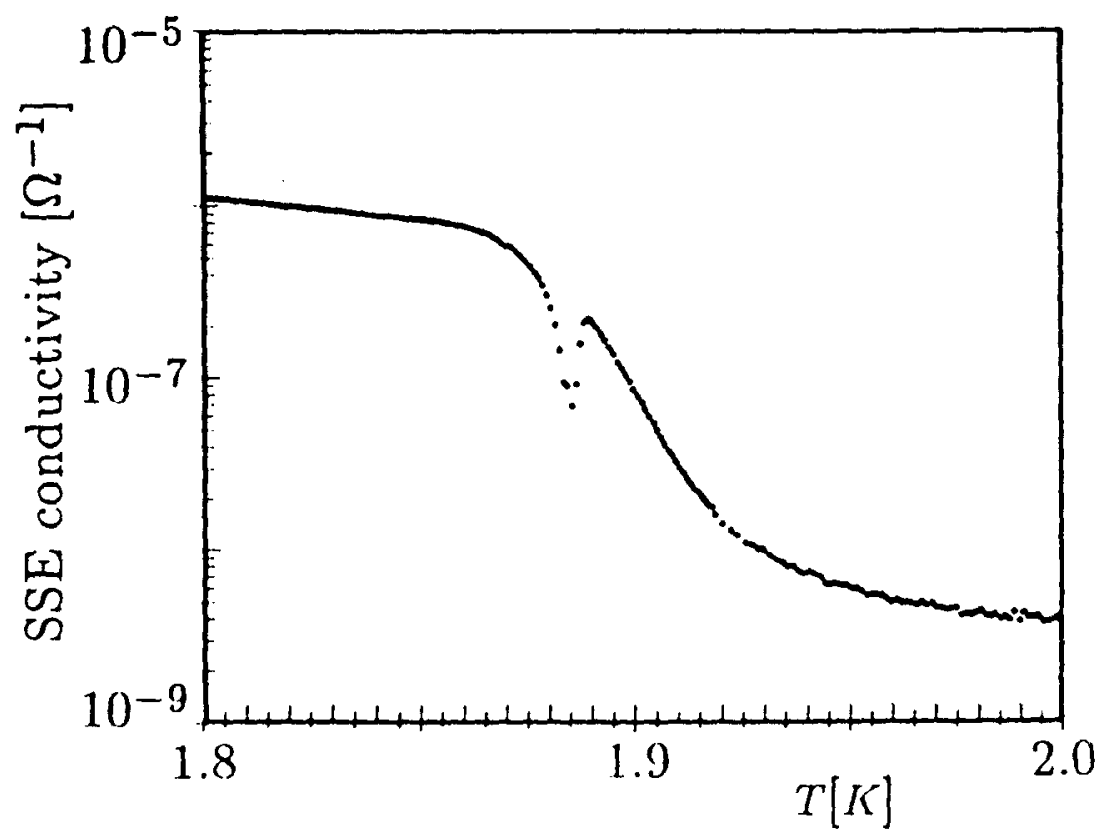

Fig. 5. Conductivity of SSE on a ${ }^{4} \mathrm{He}$ film in the transition region from a monolayer to a thick saturated film. The dip occurs at a film thickness of $35 \AA$, as estimated from measurements with a quartz balance (ref. 16).

but also for He films on solid $\mathrm{H}_{2}$ grown from the bulk liquid, ${ }^{13,18} \mathrm{He}$ films on solid $\mathrm{Ne},{ }^{17}$ and even for He films on sapphire ${ }^{24}$ (although in the latter case the dip occurred at a much larger film thickness).

As to now a conclusive interpretation for the mobility dip apparently does not yet exist. Andrei ${ }^{24}$ and Paalanen and Iye $^{18}$ have ascribed it to electron ripplon scattering, which is small for very thin and very thick films and has a maximum at a film thickness near the value where the dip is observed. However, at a temperature around $2 \mathrm{~K}$ this contribution ought to be rather weak, and it is not clear why it should lead to such a sharp structure. A resonance enhancement can also be excluded, because in the investigations of Kono et al. ${ }^{16}$ the dip was found to be not affected at all when the frequency of the ac conductivity measurement was varied between 1 and $100 \mathrm{kHz}$. As another source for the dip one might consider the formation of a polaronic state, which has been discussed quite extensively in the literature: ${ }^{25-29}$ Under the influence of the image and external electric fields an electron exerts a pressure upon the film surface, and thus can produce a dimple in the He film underneath it. The mobility of this polaron 
(=electron + dimple) will be drastically reduced compared with the quasifree electrons. Estimates show that for $\mathrm{He}$ films on $\mathrm{H}_{2}$ the energy gain on forming a polaron is indeed largest for a film thickness of about 10 monolayers. ${ }^{16}$ Although this energy is smaller than $k_{B} T$ for the experiments discussed here, the tendency to form a dimple could give rise to precursor

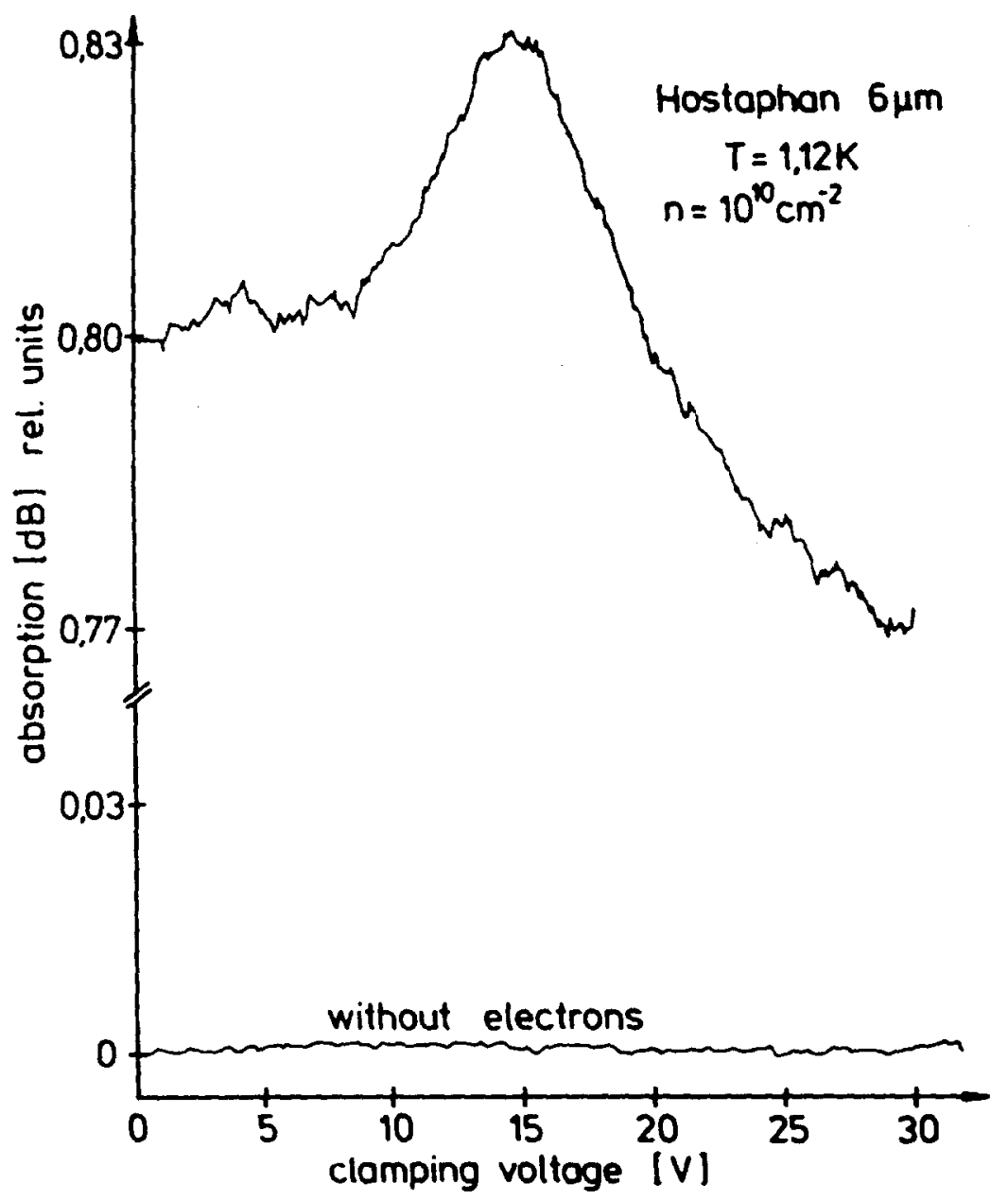

Fig. 6. The microwave absorption of electrons on a helium film at a frequency of $9 \mathrm{GHz}$ and a temperature of $1.12 \mathrm{~K}$ as a function of the applied clamping voltage. A polymer foil, $6 \mu \mathrm{m}$ thick, of Hostaphan (Mylar) served as a substrate for the helium film (thickness $d=150 \AA$ ). The electron density was about $2 \times 10^{10} \mathrm{~cm}^{-2}$ (top curve). A run without electrons (bottom curve) is shown for comparison (taken from ref. 35 ). 
effects similar to the soft mode observed near the macroscopic dimple instability. $^{7}$

It should be added that for He films on classical substrates a sharp drop with decreasing film thickness, qualitatively similar to the one in Fig. 5 , has been observed, which has been interpreted as a transition from a delocalized to a polaron state. ${ }^{24}$ Present theories, on the other hand, predict that polarons on a perfectly smooth substrate should develop only at temperatures substantially lower than the range of the experiments. Apparently pinning by surface roughness plays an important additional role for the localization of the electrons. ${ }^{30}$

\section{Saturated He Films}

The mobility of SSE on saturated He films is in general extremely low (except, as discussed, for $\mathrm{H}_{2}$ and $\mathrm{Ne}$ ) due to pinning at surface irregularities. It could be shown by Yiang and $\mathrm{Dahm}^{34}$ that the degree of pinning was substantially reduced and the mobility therefore increased when using a very smooth surface, like float glass, which in addition was carefully cleaned. With such a substrate these authors studied the sliding of the SSE at high density, where a 2-D electron solid is formed, and found features in the conductivity and noise characteristics similar to sliding charge density waves.

Electronic transport studies on saturated films yielded also another interesting result, shown in Fig. 6: Lehndorff and Dransfeld ${ }^{35}$ measured the microwave absorption of SSE at a frequency of $9 \mathrm{GHz}$ and a temperature $T \geq 1 \mathrm{~K}$ and found a broad maximum as a function of the external holding field. It was suggested that with the mylar substrate used in this experiment polarons were formed on the saturated $\mathrm{He}$ film, and that the absorption maximum reflected the lateral resonance vibration of the electrons localized in their dimples. Further investigations of this phenomenon for variable film thickness, magnetic field and lower temperature should give valuable information about the polaron state.

\subsection{Electrons at the ${ }^{4} \mathrm{He}$ Solid-Liquid Interface}

The interface between solid and superfluid ${ }^{4} \mathrm{He}$ provides a very suitable system to investigate crystal growth, because

-both atomically smooth and rough interfaces can be studied readily, and the transition between these two states at a "roughening temperature" $T_{R}$-which for the basal plane of $h c p{ }^{4} \mathrm{He}$ is at $1.3 \mathrm{~K}^{41,42}$-is easily accessible;

- the latent heat of melting is small over a wide temperature range, as is obvious from the nearly vanishing slope of the melting curve; 
-the thermal conductivity of the superfluid ${ }^{4} \mathrm{He}$ phase is extremely high.

The latter two properties greatly reduce the usual complications caused by heat transport in the bulk materials, so that the crystal growth in this case is dominated by the intrinsic processes at the interface. As a result the relaxation of the solid-liquid interface can become extremely fast, giving rise to intriguing new phenomena such as melting-crystallization waves and a highly anomalous boundary resistance for the transmission of phonons.

Electrons can be used in the form of electron bubbles to study the dynamics of the solid-liquid interface of helium in the following way:

When bubbles are pulled towards the crystal-liquid interface by means of an externally applied electric field $E$, they are trapped at the interface because this boundary presents an energy barrier of several hundred $K$, large compared to the thermal energy. ${ }^{46}$ At a typical bubble density of about $10^{8} \mathrm{~cm}^{-2}$ used in the experiments the average interparticle spacing is $\sim 10^{4} \AA$, so that a noticeable change of the interfacial properties due to the bubbles is not expected. Since the bubbles exert a small but finite pressure upon the interface under the influence of the external field, a thin layer of the crystal melts. This at the first sight somewhat surprising observation is due to the fact that the chemical potential of solid is increased, since the electronic pressure acts on the solid phase (which presents the potential barrier for the charges), whereas the chemical potential of the liquid is not altered by the charges. Consequently the energy of the system is lowered when a certain portion of the solid is transformed into the liquid state. Equilibrium is attained at a depth $\zeta_{0}$, where the electrostatic pressure is balanced by the hydrostatic pressure, i.e. when

$$
\Delta \rho g \zeta_{0}=P_{e l}
$$

Here $\Delta \rho$ is the density difference between the liquid and solid phase. Effects of the interfacial tension have been neglected in (1), because we are studying deformations of the interface on a scale large compared to the capillary length (which is about $1 \mathrm{~mm}$ for the hcp-superfluid ${ }^{4} \mathrm{He}$ interface). ${ }^{7}$ Since the heat produced with our technique is exceedingly small, temperature differences can be neglected as well.

The new equilibrium position of the crystal surface is reached with a characteristic relaxation time $\tau$, which is a measure of the speed of melting. Similarly, information about the growth is obtained from the relaxation back to the original crystal position as the electric field is reduced to the starting conditions. The motion of the interface can be measured, for example, by an interferometric technique, which provides a resolution of changes of height as small as $0.5 \mu \mathrm{m}$. This allows one to measure the 
behavior of crystal growth even for extremely small deviations $\Delta p$ from the equilibrium pressure $p_{m}$ at the melting curve (the ratio $\Delta p / p_{m}$ can be as small as $10^{-10}$ ).

The response of an atomically rough interface to momentary changes of the electrostatic pressure is shown in Fig. 7. The relaxation of the interface into the corresponding new equilibrium states, plotted in Fig. 8 on a semilogarithmic scale, closely follows an exponential in this case:

$$
\zeta=\zeta_{0} e^{-t / \tau}
$$

Equation (2) can also be written as

$$
\dot{\zeta}=-\frac{1}{\tau} \zeta
$$

Since $\dot{\zeta}$ is the growth velocity $v$, and $\zeta=\Delta \mu \rho_{c} / g \Delta \rho$, we have

$$
v=-\left(\frac{\rho_{c}}{g \Delta \rho \tau}\right) \Delta \mu
$$

where $\rho_{\mathrm{c}}$ is the density of the crystal.

Thus the relaxation time $\tau$ measured in the experiment is related to the kinetic growth coefficient $\left(m_{4} K\right)$, defined by $v=-\left(m_{4} K\right) \Delta \mu$, by

$$
\tau^{-1}=\left(m_{4} K\right) g \Delta \rho / \rho_{c}
$$

According to Eq. (3) the growth velocity is proportional to $\Delta \mu$ as expected for an atomically rough surface. But what are the processes which limit the crystal growth in the case of ${ }^{4} \mathrm{He}$ ? A clue can be obtained by studying the temperature dependence of the relaxation time. Results for hcp ${ }^{4} \mathrm{He}$ crystals with two different orientations are plotted in Fig. 9. Obviously the relaxation of the crystal surface towards the equilibrium position develops faster as the temperature is lowered. This rules out thermally activated processes such as nucleation to dominate crystallization in this case, because these would lead to just the opposite temperature dependence. The straight line in Fig. 9 suggests a relation

$$
\tau \sim\left(m_{4} K\right)^{-1} \sim e^{-\Delta E / k_{B} T}
$$

(The slight temperature dependences of $\Delta \rho$ and $\rho_{c}$, which according to Eq. (4) enter the relation between $\tau$ and $\left(m_{4} K\right)^{-1}$, can be neglected here.) Data for other crystal surfaces show the same behavior, with $\Delta E / k_{B}=7.6 \pm 0.5 \mathrm{~K}$, irrespective of the orientation. An exception are $\{0001\}$-surfaces below about $1.3 \mathrm{~K}$ as seen from the open symbols in Fig. 9. The drastic increase of the inverse growth coefficient and hence of the relaxation time on lowering the temperature is apparently related to the roughening transition of the basal plane. 


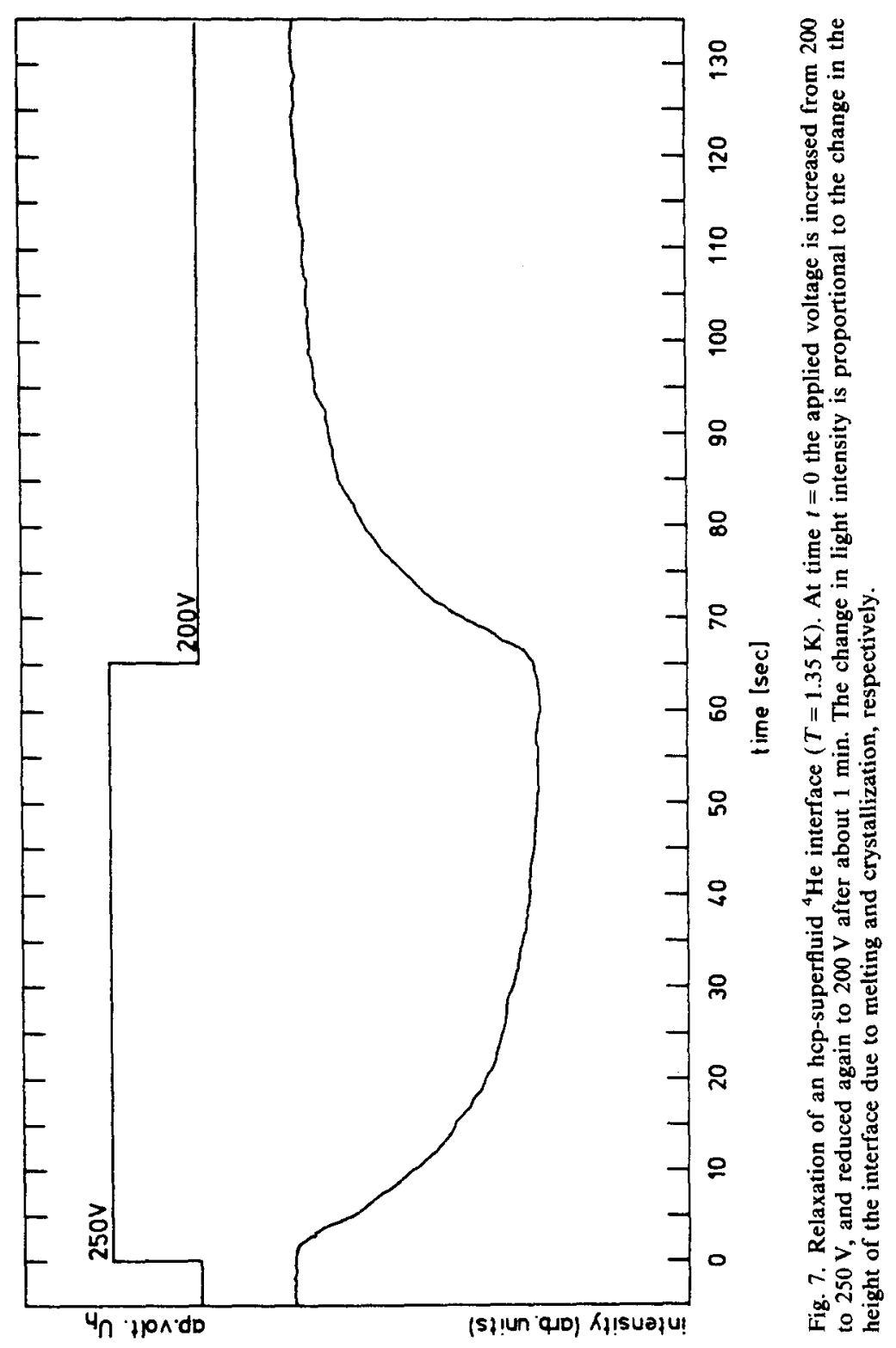




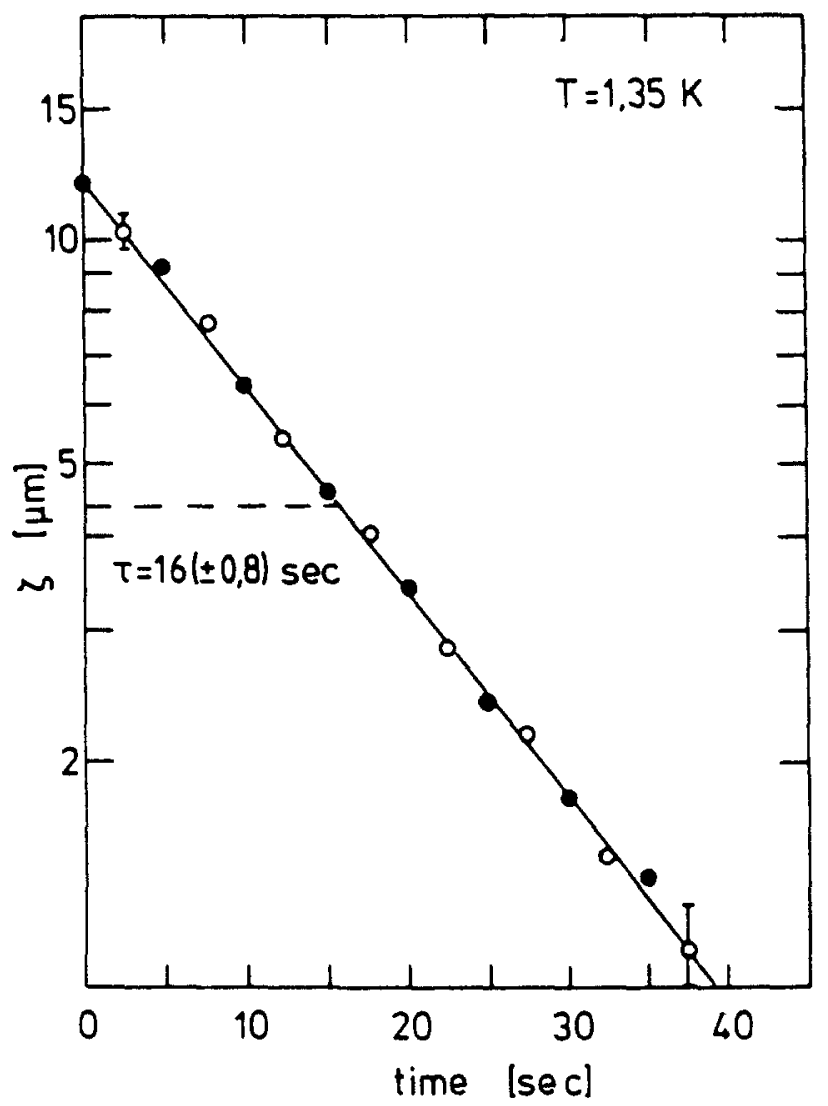

Fig. 8. Position of the crystal-superfluid interface versus time as derived from the relaxation process in Fig. 7. Open symbols refer to growth, and closed symbols to melting. The relaxation times for both processes are the same, as expected when the deviations from equilibrium are small.

The observed dependence of the growth coefficient on temperature is in very good agreement with data at lower temperatures, obtained by other groups from melting-crystallization waves ${ }^{43}$ and sound transmissions ${ }^{44,47}$ through the interface. As suggested already in the work of Keshishev et $a l^{43}$ the growth in ${ }^{4} \mathrm{He}$ at low temperatures appears to be dominated by phonons and rotons. The observed dependence (5) therefore agrees very well with this picture, because a relation similar to (5) with the same $\Delta E$ governs the number of rotons in liquid ${ }^{4} \mathrm{He}$ (in the temperature range investigated here, the rotons by far dominate over the phonons). At temperatures above $1.45 \mathrm{~K}$, where the ${ }^{4} \mathrm{He}$ crystals along the melting curve have 


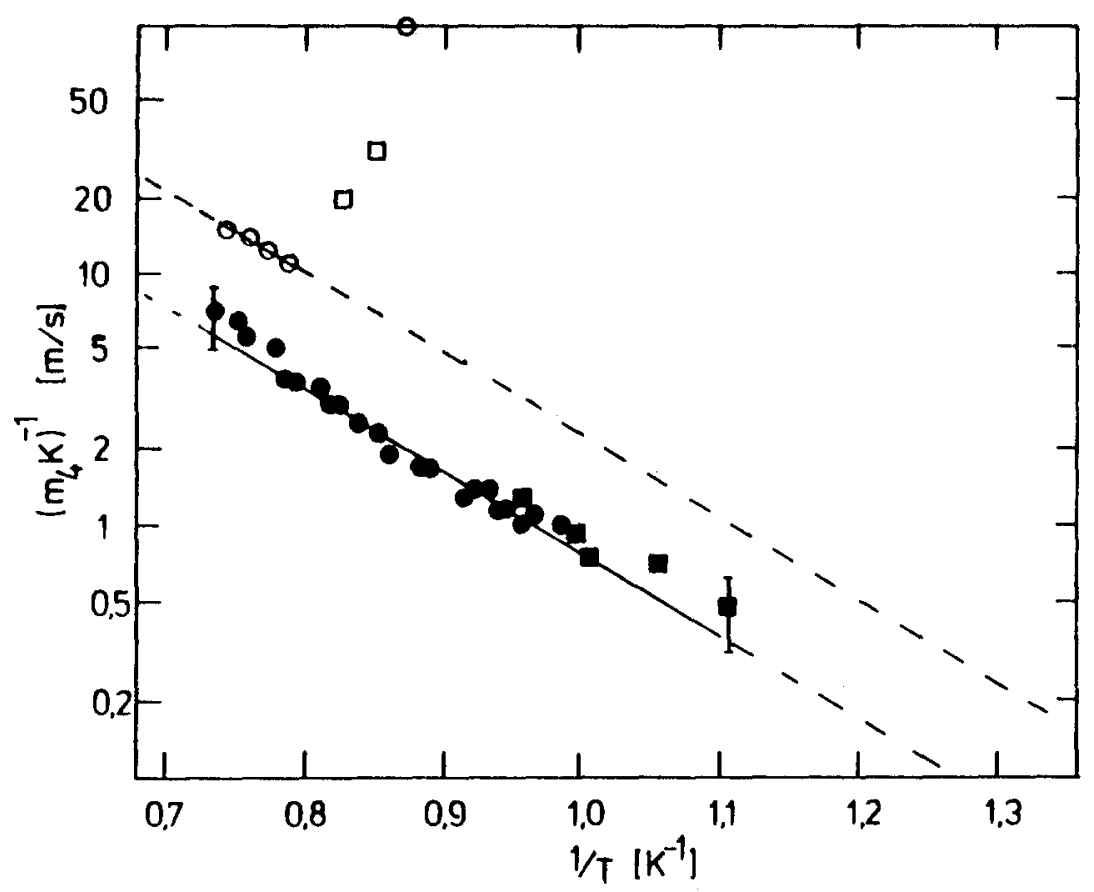

Fig. 9. Inverse growth coefficients of several hcp crystals with [0001] (open symbols) and [1010] (closed symbols) orientation. Relaxation times for intermediate crystal orientation were found to lie between the two straight lines. The slopes in this case corresponds to $\Delta E / k_{B}=7.3 \mathrm{~K}$. Data for the (0001) plane below $1.25 \mathrm{~K}$ refer to initial slopes of the relaxation curves and are only intended to give an impression of the time scale of crystal growth, because the relaxation process in this range is no longer exponential.

bcc structure, relaxation times for the liquid-solid interface were found which in their absolute value extrapolate from the HCP data, but in their temperature dependence show a small, yet significant, deviation from the simple exponential given by (5). This could be ascribed to the temperature dependence of the roton energy in this range, $\Delta E$ being reduced as $T$ is raised.

The first theories relating the growth coefficient to the thermal excitations in the bulk phases, as presented by Andreev and Knizhnik ${ }^{48}$ and Bowley and Edwards, ${ }^{49}$ predicted a behavior as given by (5) only for the "ballistic regime" at low temperatures where the mean free path of the excitations is large. The measurements around $1 \mathrm{~K}$ discussed here, however, refer to the "hydrodynamic regime" where a nearly constant growth coefficient was expected. In a more recent work by Edwards et al. ${ }^{50}$ this 
deficiency was removed, so that now the agreement between theory and experiment is quite satisfactory.

\section{INTERACTION BETWEEN THE ELECTRONS AND INTERFACIAL EXCITATION: THE EHD INSTABILITY}

The excitation spectrum of waves at an (uncharged) liquid-gas or a liquid-liquid interface is determinated by two contributions, due to gravity and interfacial tension:

$$
\omega_{0}^{2}=\frac{\rho_{l}-\rho_{u}}{\rho_{l}+\rho_{u}} g k+\frac{\sigma_{i}}{\rho_{l} \rho_{u}} k^{3}
$$

Here $\omega_{0}$ and $k$ are the frequency and wave vector of the excitation, $g$ is the acceleration due to gravity, $\rho_{u}$ and $\rho_{l}$ are the densities of the upper and lower phase, and $\sigma_{i}$ is the interfacial tension. In Eq. (6) it is assumed that the depth of the liquid is large compared with $1 / k$, and that the dissipation is negligible. For long wavelengths, the first term on the rhs of Eq. (6) dominates, giving rise to the gravitational waves with a dispersion relation $\omega_{0} \sim k^{1 / 2}$. The capillary waves at short wavelengths, on the other hand, obey the relation $\omega_{0} \sim k^{3 / 2}$. The transition between the two regimes occurs around a characteristic wavelength $\lambda_{c}=2 \pi a$, where $a=(\rho g / \sigma)^{1 / 2}$ is the so-called capillary length, which is typically on the order of millimetres. The resulting dispersion relation for the interface of phase separated liquid ${ }^{3} \mathrm{He}-{ }^{4} \mathrm{He}$ mixtures is shown as a solid line in Fig. 10.

When the interface is charged with electrons (or positive or negative ions, respectively), an additional contribution has to be taken into account, which reduces the frequency of the excitations ${ }^{51,52}$ :

$$
\omega^{2}=\omega_{0}^{2}-\frac{E_{l}^{2}+E_{u}^{2}}{4 \pi\left(\rho_{l}+\rho_{u}\right)} k^{2}
$$

$E_{l}$ and $E_{u}$ are the electric fields below and above the interface (related to the 2-D charge density $\rho$ by $E_{u}-E_{1}=2 \pi \rho$ ), and we have assumed that the charge carrier mobility is high enough so that the interface is always an equipotential. The physical origin for the reduction in $\omega$ is given by the fact that a local elongation of the surface leads to a redistribution of charges and consequently to a nonuniform electrostatic force which counteracts the restoring forces due to gravity and interfacial tension.

Equation (7) is interesting because not only does it predict a softening of interfacial waves, but also an instability of the interface when a critical electric field (or a critical charge density) is reached. The condensed phases of the helium isotopes, which provide liquid-gas, liquid-liquid, and liquidsolid interfaces, have proved to be a unique system for studying these effects of charges below, near, and above the instability threshold. 


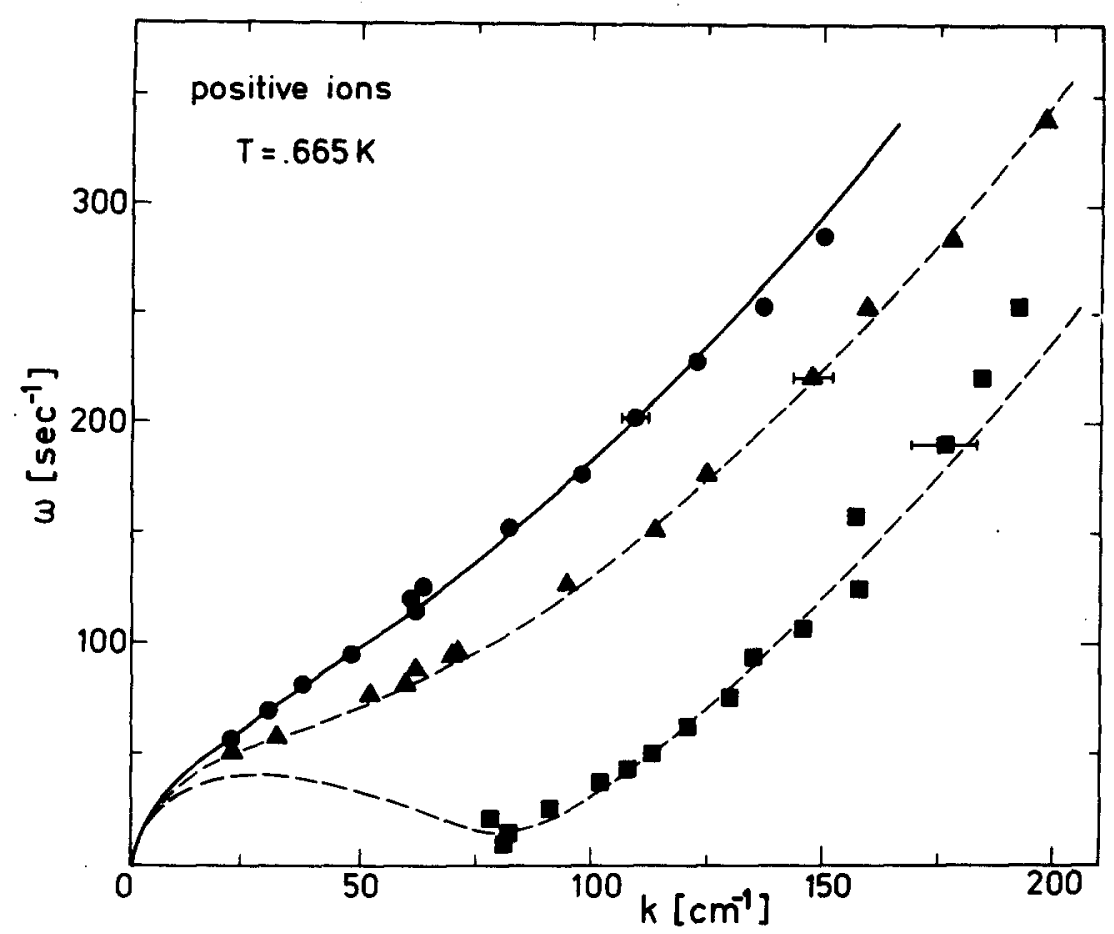

Fig. 10. Dispersion relation of ripplons at the interface of aphase separated ${ }^{3} \mathrm{He}-{ }^{4} \mathrm{He}$ mixture completely charged with positive ions. The interface becomes unstable at $E_{c}=875 \mathrm{~V} / \mathrm{cm}$, corresponding to an ion density $n_{c}=4.8 \times 10^{8} \mathrm{~cm}^{-2} .0, E / E_{c}=0.12 ; \Delta, 0.71 ; \mathbf{0}, 0.995$. The dashed curves are calculated according to Eq. (7). The dispersion of the uncharged interface is given by the solid line.

\subsection{Softening of Interfacial Waves}

An example where the influence of charges on the excitation spectrum of interfacial waves (also called ripplons) has been demonstrated in some detail are phase-separated ${ }^{3} \mathrm{He}-{ }^{4} \mathrm{He}$ mixtures below the tricritical point. ${ }^{53}$ At this interface between a ${ }^{4} \mathrm{He}$-rich superfluid and a ${ }^{3} \mathrm{He}$-rich normal liquid both positive and negative ions can be trapped provided the two coexisting phases are not too similar (i.e. that one is not too close to the tricritical temperature).$^{54}$

Figure 10 shows some data, together with the predictions of Eq. (7), which illustrate the ripplon softening. The reduction of the ripplon frequency is most pronounced at wave vectors around the inverse capillary length, $1 / a$.

The softening of interfacial waves is reminiscent of structural phase transitions, where a soft mode signals that a system approaches some instability point. In fact, also for charged helium interfaces an instability 
and a transitions to a state with a new symmetry is observed, as discussed in the following section.

\subsection{Formation of the Dimple Lattice}

For an interface charged to saturation the electric field in one of the phases is screened completely by the interfacial electron or ion density $n$, while in the other it is $E=4 \pi n e$. As seen from Eq. (7) and from Fig. 10, the frequency of the soft ripplon mode at $k=1 / a=k_{c}$ vanishes when the charge density ne reaches a critical value $n_{c} e=\left[\left(\rho_{l}-\rho_{u}\right) g \sigma_{i} / 4 \pi_{2}\right]^{1 / 4}$ at a corresponding critical field $E_{c}=4 \pi_{c} e$. For $n$ just above $n_{c}, \omega$ becomes imaginary at a wave vector $k=k_{c}$, which implies that fluctuations in the elongation of the interface with that particular $k$-value will increase in time instead of decay. As a result, a disruptive loss of charge from the interface develops. ${ }^{55}$

A qualitatively quite different feature of the EHD instability is observed, when the surface is not charged to saturation, but to only about $10 \%$ of $n_{c}$ or less. ${ }^{56}$ Although again interfacial waves with $k=k_{c}$ become unstable as the electric field is raised somewhat above a critical value $E_{c}$ (and consequently the interface ripples spontaneously with a characteristic wavelength, which again is $\left.\lambda_{c}=2 \pi / a\right)$, nonlinear contributions to the restoring force stabilize these deformations at a finite amplitude. Thus a new equilibrium state in the charge distribution develops, different from the homogeneous layer of electrons for fields below $E_{c}$.

The formation of the new state typically occurs in two steps, as illustrated in Fig. 11 (in these photographs, taken by a Schlieren technique, local maxima (Fig. 2(a,b)) or minima (Fig. 2(c)) of the deformed surface appear bright). First, a standing wave-like pattern with $k=k_{c}$ develops, which then breaks up further into a periodic array of macroscopic dimples. Simultaneously the electrons, being pulled into local minima of the surface by the externally applied field, first accumulate in the wave troughs and then at the centres of the dimples. The typical duration of the whole process is a few seconds. Since the resulting triangular "dimple crystal" has developed from the soft ripplon mode, its lattice spacing is close to $\lambda_{c}$.

The transition to the dimple state is reversible: By reducing the electric field the homogeneous charge distribution and the flat interface are retained. If the charge density is lower than the above mentioned limit of $\sim 0.1 n_{c}$, the system can repetitively be cycled through the instability without a loss of charge. An analysis of this transition shows that-in terms of the language of phase transitions-it is of weakly first order. ${ }^{57,58}$ Consequently, hysteresis effects in the dimple formation are expected on varying the electric field $E$, which here plays the role of an effective temperature. Such effects have 

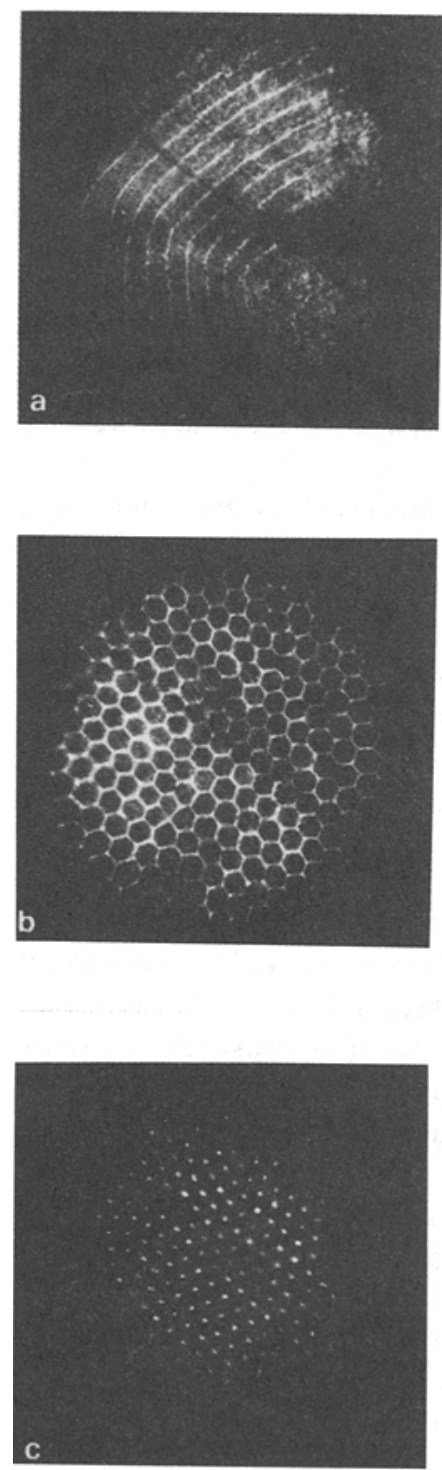

Fig. 11. Formation of a dimple lattice on a ${ }^{4} \mathrm{He}$ surface $(T=35 \mathrm{~K})$ charged with electrons from above. The pictures show the surface deformation approximately $2 s(a)$ and $6 s((b)$ and (c)) after the field had been increased to $E_{c}$. The image plane in (a) and (b) was chosen such that convex deformations of the surface, corresponding to local maxima, appear bright; in (c) bright areas correspond to local minima (i.e. the center of the dimples). The distance between adjacent rows of dimples is close to the wavelength $2 \pi a$ of the soft ripplon, $0.24 \mathrm{~cm}$ in this case. 
been observed indeed in a range/1-(E/Ec $) /$ of the order of $10^{-2} .^{56,59}$ Furthermore, in an experiment where the liquid surface was carefully shielded from external vibrations, a striped phase has been found to be stable in a certain range close to $E_{c}{ }^{59}$

Another aspect of the dimple lattice follows from a consideration of the charge distribution in this state: As numerical calculations show, ${ }^{60,61}$ the charge density is not just slightly modulated along the surface, but is large in a region close to the dimple centres and zero elsewhere. Since the potential barrier between neighboring dimples is high, the amount of charge of each dimple is fixed, and as a result the interaction between the dimples is dominated by Coulomb repulsion with only a small contribution from the deformation of the interface. The dimple lattice can therefore be regarded as an example of a 2-D Coulomb crystal, and is in many respects a macroscopic counterpart of the (microscopic) Wigner crystal introduced in Sec. 1. Lattices like the one in Fig. 11 not only have the triangular symmetry expected for such 2-D systems consisting of particles with isotropic interaction, but even display typical defects like dislocations and grain boundaries. ${ }^{59,60}$

The existence of multielectron dimples on liquid helium does not depend on the formation of a more or less regular array. If only few electrons are present at the surface and the electric field is chosen properly, dimples can develop and are stable also as single, isolated objects. Characteristic properties like width, depth, and eigenfrequencies of such individual dimples have been determined by several authors. ${ }^{58,60-63}$ Typically, a dimple containing $5 \times 10^{6}$ electrons is a few tenths of a mm deep and has a width on the order of the capillary length, which is in the mm range. It should be mentioned that dimple formation and the appearance of dimple crystals is not restricted to the free surface of liquid ${ }^{4} \mathrm{He}$, but has been observed for all the liquid-gas, liquid-liquid, and liquid-solid interfaces of condensed helium.

\subsection{The Growth of the EHD Instability}

For electric fields distinctly higher than the critical value $E_{\mathrm{c}}$ the restoring force due to the deformation is no longer sufficient to confine the charge at the interface. The resulting dynamics of the instability can be described, in its initial stage, by an extension of Eq. (7), which now in a certain band of wave vectors yields unstable solutions. The range of unstable modes is plotted in the stability diagram in Fig. 12 as a function of the reduced electric field $E / E_{c}$ :

-for $E<E_{c}$ the system is stable for all values of $k$;

-at the instability threshold $E=E_{c}$ the mode with $k=k_{c}$ becomes unstable; 


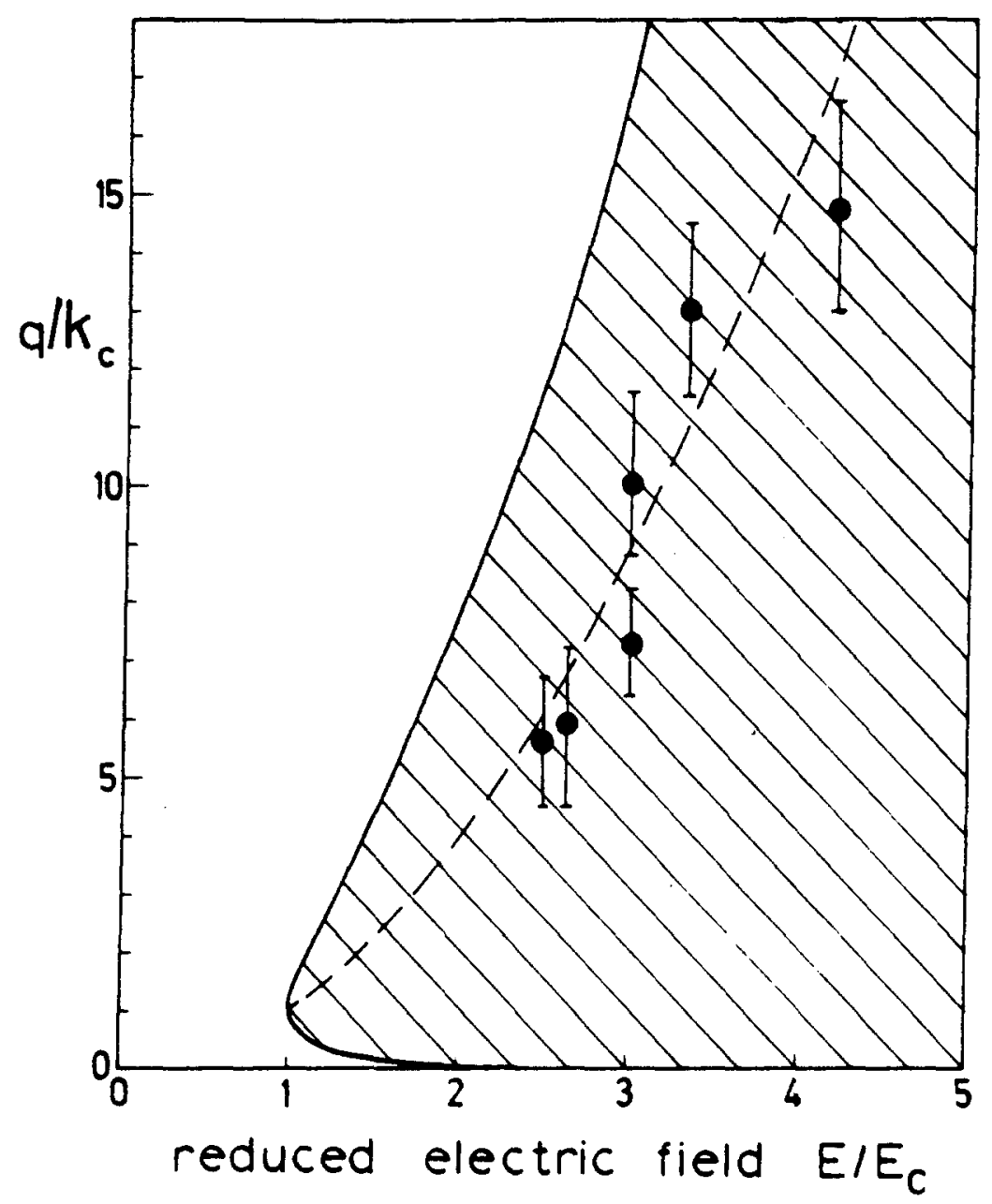

Fig. 12. Stability diagram of a charged interface, as calculated from Eq. 7. The dashed line indicates the unstable wave vectors with the largest gain. The data points represent experimental results of $\boldsymbol{m}$ for the hcp-superfluid ${ }^{4} \mathrm{He}$ interface.

-for $E>E_{c}$ the unstable range, where a perturbation grows instead of decaying, covers an increasing band of wave vectors.

In general, the "punch-through" process of the charges leads to a severe perturbation of the interface. The EHD deformations grow in amplitude, until charged bubbles split off and move to the counter electrode. For the liquid-gas interface this process, which can take place in milliseconds, has been recorded with a high speed camera. ${ }^{55}$ Since the damping of surface waves in this case is small, the perturbations add up and the whole interface 
immediately is in chaotic motion (except if the geometry is chosen properly; see Sec. 3.4). An interface which allows one to observe the development of the EHD instability under better-controlled conditions and in somewhat more detail is provided by the boundary between liquid and solid ${ }^{4} \mathrm{He}$. As shown by Andreev and Parshin ${ }^{64}$ and by Keshishev et al., ${ }^{43}$ excitations of this interface are propagating melting-crystallisation waves whose dispersion relation is quite similar to that of ripplons at a liquid surface (Eq. (6)). The attenuation of these waves depends strongly on temperature; above $1 \mathrm{~K}$ they are overdamped in the critical wave vector range around the inverse capillary length. ${ }^{46}$ When such an interface is charged with ions, the stability diagram shown in Fig. 12 should apply as before. As a technical advantage, however, in this case the perturbing influence of waves reflected from the container walls, which usually obscure the growth of the EHD instability, is suppressed because of the strong damping, and the growth of the unstable modes can easily be followed on a time scale of seconds.

A picture of such an interface between liquid and solid ${ }^{4} \mathrm{He}$, "quenched" into the unstable regime by a sudden increase of the electric field, is presented in Fig. 13. Although the pattern is not nearly as regular as the dimple lattice in Fig. 11, some periodicity in the deformation of the crystal can still be recognized. Apparently a small range within the wide band of competing

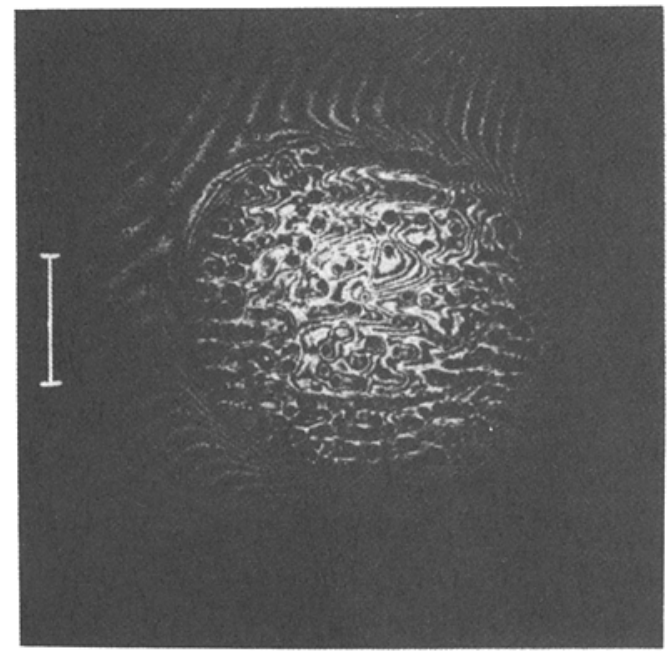

Fig. 13. Snapshot of the instability pattern developing at an hcp-superfluid ${ }^{4} \mathrm{He}$ interface at $E / E_{c}=2.6$. The characteristic distance of the stripes is distinctly smaller than the lattice spacing of the dimple crystal at the critical field, $2 \pi / k_{c}=6.3 \mathrm{~mm}$. 
unstable modes at that field dominates the growth. The orientation of the corrugations visible in Fig. 13 is related to the anisotropy of the ${ }^{4} \mathrm{He}$ crystal.

The higher the applied field the faster this instability is found to develop. Simultaneously the wave vector $k_{m}$ characterizing the spacing of the corrugations becomes larger and less well-defined. Thus there is considerable scatter in the data points plotted in the stability diagram (Fig. 12). Nevertheless the agreement with the predicted curve for the modes with the fastest growth, shown as the dashed line in Fig. 12, appears reasonable, indicating that the essential features of the phenomenon are covered by the theory sketched above.

The development of the EHD instability resembles closely, in certain respects, the phenomenon of spinodal decomposition in two-component liquids or alloys with a miscibility gap. When such a system is quenched from the one-phase state through the critical point into the unstable range, it starts to phase-separate spontaneously. This process is characterized by the instability of a whole range of wave vectors, but as for the EHD instability a particular $k$-value grows most quickly and dominates the decomposition, resulting in patterns similar to Fig. 13 . The charge-induced instability at helium interfaces thus can be considered in the context of a much wider class of critical phenomena.

\subsection{Multielectron Bubbles}

As already mentioned in Sec. 3.2, the final stage of the EHD instability involves charged bubbles which break away from the interface and move towards the counter electrode. These bubbles (filled with gas or liquid, depending on the system to be studied) are stabilized by the interfacial tension $\sigma$ and the hydrostatic pressure $p$ in the surrounding phase, counterbalanced by the repulsive Coulomb forces of the electrons at the inside of the bubble. The equilibrium bubble radius is determined by minimizing the energy ${ }^{65,66}$

$$
E=4 \pi R^{2} \sigma+\frac{4}{3} \pi R^{3} p+\frac{Z^{2} e^{2}}{(2 \varepsilon R)}
$$

(accounting for surface energy, external pressure and electrostatic energy, respectively; $\varepsilon$ is the dielectric constant and $Z e$ the total charge of the bubble). At a typical $Z$ between $10^{5}$ and $10^{7} e$, Eq. (8) yields a diameter of 10 and $100 \mu \mathrm{m}$, respectively, for gas bubbles in liquid ${ }^{4} \mathrm{He}$.

These bubbles appear as interesting objects, because due to their mutual Coulomb repulsion the charges are confined to a thin "skin" close to the bubble surface and thus constitute, on a local scale, a 2-D Coulomb system like the electrons on the flat liquid surface, but with a considerably higher 
density $\left(10^{10} \mathrm{e} / \mathrm{cm}^{2}\right.$ and more). They therefore might provide an example where quantum corrections to the otherwise classical behavior of the 2-D electron system at helium interfaces should become important. This influence should show up, e.g., in the phase diagram of the Wigner crystal.

It has been demonstrated that it is possible to generate the bubbles in a controlled manner, and to study them by means of light scattering. ${ }^{67}$ Fig. 14 shows the current pulses due to a sequence of bubbles, $10^{6}$ electrons each, which was registered by means of an electrometer.

Using proper illumination these bubbles appear as spots with sufficient intensity to record their trace with a video camera. An example is shown in Fig. 15, where the illuminating laser beam was chopped in order to determinate the bubble velocity $v$. It was found that $v$ reaches a constant value, typically $10 \mathrm{~cm} / \mathrm{sec}$ under the conditions of Fig. 15, nearly instantaneously after the bubble has formed. This implies that the energy which the moving charges pick up in the applied electric field is quickly dissipated into other channels. One of them appears to be the excitation of bubble oscillation, which is supported by the observation that the brightness along the bubble paths fluctuates strongly even if the laser intensity is kept constant. The observed oscillation frequencies are of the order of $2 \mathrm{kHz}$, which is in good agreement with calculated values for the lowest bubble mode. ${ }^{65}$

A promising aspect for further investigations comes from an experiment which has demonstrated that capture and storage of the bubble in an electric quadrupole configuration similar to an if trap for ions should be feasible. ${ }^{67}$ This would enable one to study the eigenmodes of the bubbles in much

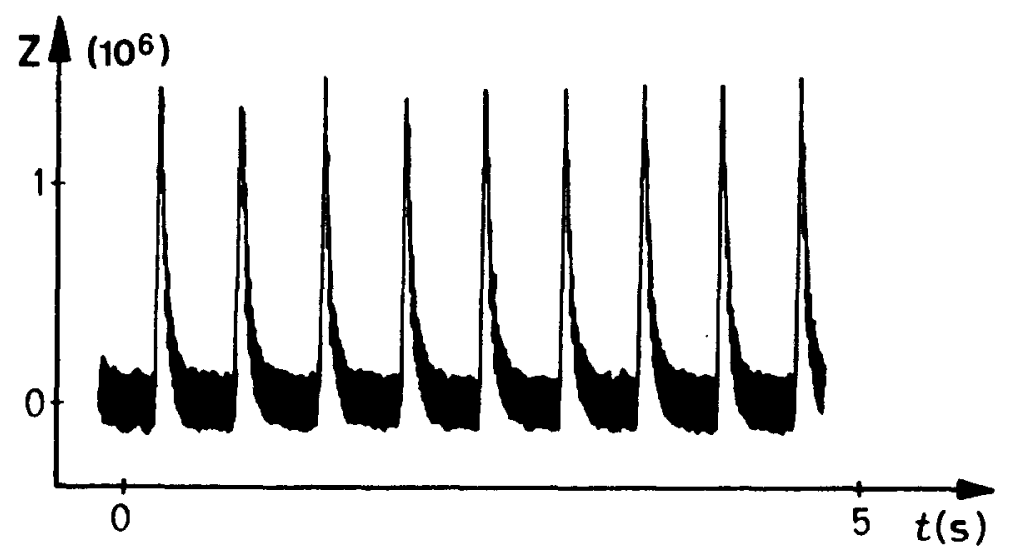

Fig. 14. Displacement current at the anode recorded at a vapor pressure of 1000 mbar. Each peak corresponds to one multielectron bubble; the peak height is proportional to the charge of the bubble ( $Z$ : number of electrons in a bubble). 


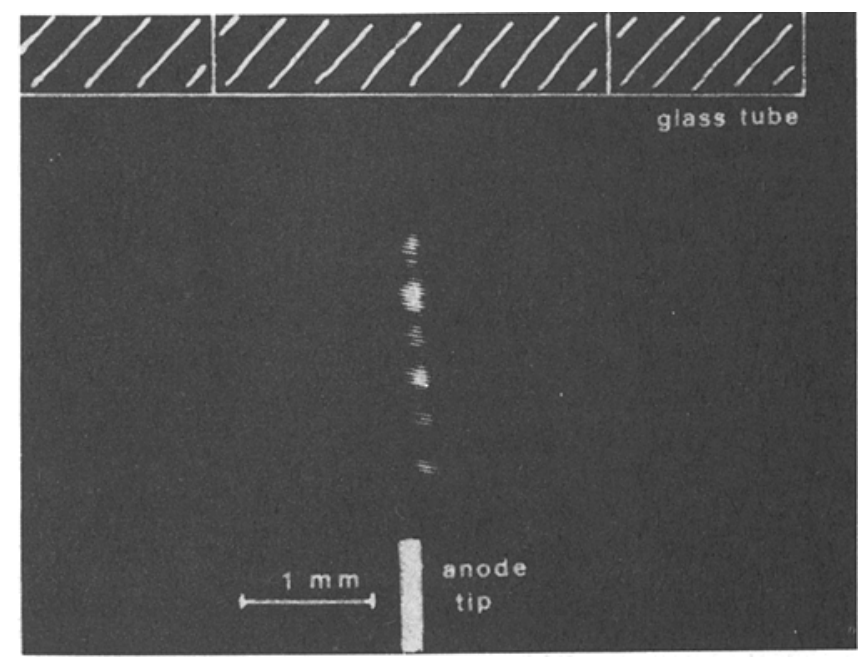

Fig. 15. Track of a single multielectron bubble moving towards the anode tip. The irradiating laser is chopped at a frequency of $370 \mathrm{~Hz}$ to measure the velocity of the bubble. The size of the spots is not the size of the bubble but only a measure for the intensity of the scattered light.

more detail. Of particular interest in this context is the influence of the bubble surface charge on this mode, which is expected to reflect changes in the 2-D electron system, like the transition to the Wigner crystal at high electron densities.

\subsection{Electrons on Helium Films}

An alternative route for for reaching electron densities in excess of the limit imposed by the EHD instability of the bulk surface is to use a helium film as a substrate. The stability of a film is increased due to van der Waals forces, as seen from the dispersion relation for ripplons coupled to the 2-D electrons layer:

$$
\omega^{2}=\frac{\rho_{s}}{\rho}\left\{\left(\frac{3 \alpha}{\rho d^{4}}+g\right) k+\frac{\sigma}{\rho} k^{3}-\frac{4 \pi n^{2} e^{2}}{\rho} F(k, \varepsilon)\right\} \tanh (k d)
$$

Compared with the dispersion relation for a charged bulk helium surface, Eq. (7), there is an additional term which includes the van der Waals constant $\alpha$. The last factor on the rhs of Eq. (9) arises from the finite depth $d$ of the liquid film, the function $F(k, \varepsilon)$ takes the effect of image charges in the solid substrate into account, and the factor $\rho_{s} / \rho$ indicates that in a thin film only the superfluid component can move. 
According to Eq. (9) charging a film reduces the ripplon frequency in a similar way as for bulk helium, but the critical charge density where the instability occurs is higher. For a thickness of $300 \AA-a$ typical value for a saturated film at a height $H=1 \mathrm{~cm}$ above the bath level-it is expected that an electron density $n_{c}=3 \times 10^{10} \mathrm{~cm}^{-2}$ should be stable, one order of magnitude more than on bulk He.

It was shown experimentally that not only is it possible to reach this limit, but that it can even be exceeded considerably. ${ }^{31}$ It was found, for example, that saturated helium films on a hostaphan polymer foil could be charged with electrons up to a density of $0.98 \times 10^{11} \mathrm{~cm}^{-2}$ before breakthrough to the solid substrate set in. Moreover, this instability threshold appeared to be independent of the thickness $d_{0}$ of the uncharged films in the investigated range $200 \AA<d_{0}<400 \AA$.

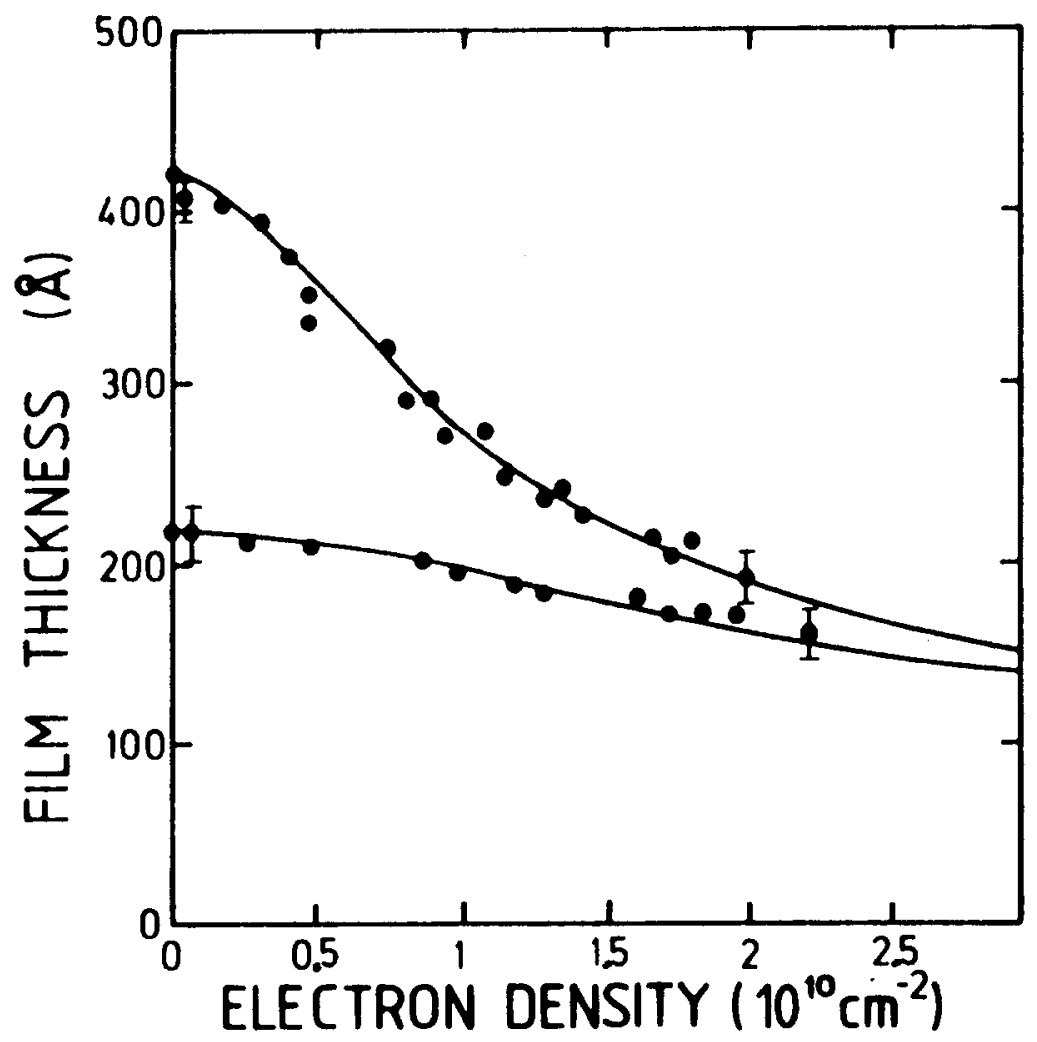

Fig. 16. Thickness $d$ of charged saturated ${ }^{4} \mathrm{He}$ films wetting a glass substrate ( $T=1.6 \mathrm{~K}$ ) versus the electron density on the films. The thickness $d_{0}$ of the uncharged films was 220 and $420 \AA$, respectively. 
This apparent discrepancy was resolved when the electronic pressure $p_{\mathrm{el}}$ exerted on the film was taken into account. Since $p_{\mathrm{el}}$ adds to the gravitational and van der Waals terms in the chemical potential, the equilibrium thickness of a charged film will be reduced to

$$
d=d_{0}\left(1+\frac{2 \pi n^{2} e^{2}}{\rho g H}\right)^{-1 / 3}
$$

assuming that the thickness before charging is given by $d_{0}=(\alpha / \rho g H)^{1 / 3}$ (retardation effects are neglected here). The value of $d$ given by Eq. (10) is an average, around which the local film thickness is expected to vary because of the formation of a simple underneath each electron. ${ }^{24-29}$ As a result of the reduction of $d$ the instability threshold, calculated from Eq. (9), is raised, and a higher charge density can be obtained.

The change in film thickness on charging was confirmed experimentally by an ellipsometric determination of $d .^{31}$ Some data are plotted in Fig. 16 for two values of the initial thickness $d_{0}$. As expected from Eq. (10) the two curves converge for high electron densities. Consequently the stability limit for high electron densities on helium films is not significantly influenced by the initial thickness, as observed in the experiment.

What limits then the charge density on helium films? Eventually, when the film has become very thin $(d \leq 50 \AA)$, tunneling of the electrons through the helium into surface states of the substrate will set in. This process might have been of importance for the highest densities reported so far, in particular since substrate inhomogeneities like surface roughness will lead to a locally reduced film thickness where tunneling can preferentially occur.

\section{CONCLUSIONS}

The various examples show that surface state electrons on hydrogen and helium are a very sensitive probe in order to study surface properties. The interaction of the electrons with these surfaces is on one hand sufficiently weak to provide a conductivity signal which can conveniently be measured, on the other hand the coupling is strong enough to give rise to large effects. The discussion has also shown that still considerable experimental and theoretical work has to be done towards a full understanding of the observed effects. Prospects for obtaining more quantitative results about quantum surfaces, possibly by a combination of SSE with other techniques, appear promising.

As pointed out in the beginning, this survey has focused on effects related to the interaction of SSE with surface structures and excitations. Two-dimensional electron systems are of course of much more general interest. Recently the value of $\mathrm{He}$ and $\mathrm{H}_{2}$ substrates for investigations of 
phenomena like magnetotransport and localization in 2-D has been demonstrated, ${ }^{36-39}$ and many more suggestions for experiments with surface state electrons exist. ${ }^{40}$

\section{ACKNOWLEDGMENTS}

In the course of this work I have greatly benefited from a close cooperation with $\mathrm{K}$. Kono und $\mathrm{U}$. Albrecht, and from numerous discussions with D. Cieslikowski, A. J. Dahm and V. B. Shikin. Support by the Deutsche Forschungsgemeinschaft, SFB 306, is also gratefully acknowledged.

\section{REFERENCES}

1. See, e.g., J. G. Dash, Phase transitions at interfaces: Roughening, surface melting and triple point wetting, Solvay Conference on Surface Science (University of Texas, 1987).

2. H. J. Lauter, Inelastic neutron scattering from adsorbates, Phonons 89 , S. Hunklinger, W. Ludwig, and G. Weiss, eds. (World Scientific, Singapore, 1990), p. 871.

3. M. W. Cole, Rev. Mod. Phys. 46, 451 (1974); C. C. Grimes, Surf. Sci. 73, 379 (1978); V. S. Edel'man, Sov. Phys. Usp. 23, 227 (1980); F. I. B. Williams, Surf. Sci. 113, 371 (1982); Yu. P. Monarkha and V. B. Shikin, Sov. J. Low Temp. Phys. 8, 279 (1982); N. Studart and O. Hipolito, Revesta Brasileira de Fisica 16, 194 (1986); V. B. Shikin and Yu. P. Monarkha, "Two-dimensional charged systems in liquid helium," Editorial Board for Physical and Mathematical Literature (Moscow, 1989).

4. V. V. Zav'yalov and I. I. Smol'yaninov, Sov. Phys.-JETP 65, 194 (1987).

5. C. C. Grimes and G. Adams, Phys. Rev. Lett. 42, 795 (1979).

6. P. Leiderer and M. Wanner, Surf. Sci. 98, 68 (1980).

7. P. Leiderer, Physica B 126, 92 (1984).

8. J. Bodensohn, P. Leiderer, and D. Savignac in Phonon Scattering in Condensed Matter, W. Eisenmenger, K. Lassmann and S. Döttinger, eds. (Springer, 1984), p. 266.

9. J. Bodensohn, K. Nicolai, and P. Leiderer, Z. Phys, B 64, 55 (1986).

10. A. M. Troyanovskii, A. P. Volodin, and M. S. Khaikin, JETP Lett. 29, 382 (1979).

11. A. M. Troyanovskii and M. S. Khaikin, Sov. Phys.-JETP 54, 214 (1981).

12. V. S. Edel'man and M. I. Faley, J. Low Temp. Phys. 52, 301 (1983).

13. D. Cieslikowski, P. Leiderer, and A. J. Dahm, Can. J. Phys. 65, 1525 (1987).

14. D. Cieslikowski, A. J. Dahm, and P. Leiderer, Phys. Rev. 58, 1751 (1987).

15. J. L. Levine and T. M. Sanders, Phys. Rev. 154, 138 (1967).

16. K. Kono, U. Albrecht and P. Leiderer, J. Low Temp. Phys. 82, 279 (1991); U. Albrecht, H. Dilger, P. Leiderer, and K. Kono, Physica B 165 \& 166, 841 (1990).

17. K. Kajita, Jap. J. of Appl. Phys. 26-3, 1943 (1987).

18. M. A. Paalanen and Y. Iye, Phys. Rev. Lett. 55, 1761 (1985).

19. S. E. Polanco, J. H. Quateman, and M. Bretz, J. Phys. (Paris), Colloq. 39, C6-344 (1978).

20. J. D. Maynard and M. H. W. Chan, Physica B 109 \& 110, 2090 (1982).

21. P. A. Shirron, K. A. Gillis, and J. M. Mochel, J. Low Temp. Phys. 78, 157 (1990).

22. G. Mistura, G. Zimmerli, and M. H. W. Chan, Study of a Layered Superfluid Film, poster presented at the Nato ASI "Phase Transitions in Surface Films" (Erice 1990).

23. E. Krotscheck, Phys. Rev. B 32, 5713 (1985).

24. E. Y. Andrei, Phys. Rev. Lett. 52, 1449 (1984).

25. L. M. Sander, Phys. Rev. B 11, 4350 (1975).

26. M. Saitoh, J. Phys. C: Solid State 16, 6995 (1983).

27. F. M. Peeters and S. A. Jackson, Phys. Rev. B 31, 7098 (1985).

28. M. H. Degani and O. Hipolito, Phys. Rev. B 32, 3300 (1985).

29. F. M. Peeters and S. A. Jackson, Phys. Rev. B 34, 1539 (1985). 
30. Yu. Z. Kovdrya, F. F. Mende, and V. A. Nikolaenko, Sov. J. Low Temp. Phys. 10, 589 (1985).

31. H. Etz, W. Gombert, W. Idstein, and P. Leiderer, Phys. Rev. Lett. 53, 2567 (1984).

32. V. V. Tatarskii, Sov. J. Low Temp. Phys. 12, 255 (1986).

33. X. L. Hu and A. J. Dahm, Comments on the stability of charged thin helium films, to be published.

34. H. W. Jiang and A. J. Dahm, Phys. Rev. Lett. 62, 1396 (1989).

35. B. Lehndorff and K. Dransfeld, J. Phys. France 50, 2579 (1989).

36. P. W. Adams and M. Paalanen, Phys. Rev. Lett. 58, 2106 (1987).

37. P. W. Adams and M. Paalanen, Phys. Rev. Lett. 61, 451 (1988).

38. P. W. Adams and M. A. Paalanen, Phys. Rev. B 37, 3805 (1988).

39. M. J. Lea, A. O. Stone, and P. Fozooni, Europhys. Lett. 7, 641 (1988).

40. A. J. Dahm, Excitations in $2 D$ and $3 D$ Quantum Fluids A. F. G. Wyatt and H. J. Lauter, eds. (Plenum, New York, 1991) p. 539.

41. S. Balibar and B. Castaing, J. Phys. (Paris) Lett. 41, 329 (1980); P. E. Wolf, F. Gallet, S. Balibar, E. Rolley, and P. Nozières, J. Phys. (Paris) 46, 1987 (1985).

42. J. E. Avron, L. S. Belfour, C. G. Kuper, J. Landau, S. G. Lipson, and L. S. Schulman, Phys. Rev. Lett. 45, 814 (1980).

43. K. O. Keshishev, A. Ya. Parshin, and A. V. Babkin, Sov. Phys.-JETP 30, 56 (1979); Sov. Phys.-JETP 53, 362 (1981).

44. B. Castaing, S. Balibar, C. Laroche and P. Nozières, J. Phys. 41, 897 (1980).

45. T. E. Huber and H. J. Maris, Phys. Rev. Lett. 47, 1907 (1981); J. Low Temp. Phys. 48, 463 (1982); J. Low Temp. Phys. 48, 99 (1982).

46. D. Savignac and P. Leiderer, Phys. Rev. Lett. 69, 1869 (1982).

47. B. Castaing, J. Phys. (Paris) Lett. 45, 233 (1984).

48. A. F. Andreev and V. G. Knizhnik, Sov. Phys.-JETP 56, 226 (1982).

49. R. M. Bowley and D. O. Edwards, J. Phys. (Paris) 44, 723 (1983).

50. D. O. Edwards, S. Mukherjee and M. S. Pettersen, Phys. Rev. Lett. 64, 902 (1990).

51. L. P. Gorkov and D. M. Chernikova, JETP Lett. 18, 68 (1973).

52. K. Mima and H. Ikezi, Phys. Rev. B 17, 3567 (1978).

53. M. Wanner and P. Leiderer, Phys. Rev. Lett. 42, 315 (1979); P. Leiderer, Phys. Rev. B 20, 4511 (1979).

54. P. Leiderer, M. Wanner, and W. Schoepe, J. Phys. (Paris), Colloq. 39, C6-1328 (1979).

55. A. P. Volodin, M. S. Khaikin, and V. S. Edelman, JETP Lett. 26543 (1977).

56. W. Ebner and P. Leiderer, Phys. Lett. A 80, 277 (1980).

57. H. Ikezi, Phys. Rev. Lett. 42, 1688 (1979).

58. V. B. Shikin and P. Leiderer, Sov. Phys.-JETP 54, 92 (1981)

59. R. W. Gianetta and H. Ikezi, Phys. Rev. Lett. 47849 (1981).

60. P. Leiderer, W. Ebner, and V. B. Shikin, Surf. Sci. 113405 (1981).

61. V. I. Kelnikov and S. V. Meshkov, Sov. Phys.-JETP 54, 505 (1981).

62. S. S. Nazin and V. B. Shikin, Sov. Phys.-JETP 58, 310 (1983).

63. V. B. Shikin and P. Leiderer, Solid State Commun. 47, 269 (1983).

64. A. F. Andreev and A. Ya. Parshin, Sov. Phys.-JETP 48, 763 (1978).

65. G. A. Williams and M. M. Salomaa, J. Low Temp. Phys. 57539 (1984); M. M. Salomaa and G. A. Williams, Phys. Rev. Lett. 47, 1730 (1981).

66. V. B. Shikin, JETP Lett. 27, 39 (1978).

67. U. Albrecht and P. Leiderer, Europhys. Lett. 3, 705 (1987). 